\title{
Pressure oscillation and steam cavity during the condensation of a submerged steam jet
}

Quanbin Zhao, Weixiong Chen, Fang Yuan, Wei Wang, Daotong Chong*, Junjie Yan

State Key Laboratory of Multiphase Flow in Power Engineering, Xi'an Jiaotong University, Xi'an 710049, PR China

Quanbin Zhao, E-mail address: zqb8031110.com@stu.xjtu.edu.cn

Weixiong Chen, E-mail address: chenweixiong@ mail.xjtu.edu.cn

Fang Yuan, E-mail address:spiritwalkers@163.com

Wei Wang, E-mail address:wwy.023031211@ stu.xjtu.edu.cn

Daotong Chong*, E-mail address: dtchong@mail.xjtu.edu.cn

Junjie Yan, E-mail address: yanjj@ mail.xjtu.edu.cn

\section{Corresponding author}

Daotong Chong, telephone number:86-029-82665359,

E-mail address: dtchong @mail.xjtu.edu.cn

Postal address: State Key Laboratory of Multiphase Flow in Power Engineering, Xi'an Jiaotong University, Xi'an 710049, PR China. 
Abstract: Steam jet condensation is important in many industrial applications. In this work, the pressure oscillation and steam jet patterns during submerged jet condensation in quiescent water is investigated experimentally. Firstly, it is found that even at stable condensation region, the steam cavity length varies all the time and steam bubbles separate from the steam cavity periodically. With the variation of steam cavity length and separated bubble oscillation, condensation also undergoes oscillation. Along the axial direction, the pressure oscillation intensity increases first and then decreases gradually. There is an distinct pressure oscillation peak, and the peak position varies over a length-to-diameter ratio range of $X / D=2$ to $X / D=7$. The axial position of pressure oscillation peak corresponds to the end of steam cavity. Moreover, oscillation energy analysis shows that the oscillation energy generated by separated steam bubble is much higher than that generated by the steam cavity length variation. Finally, based on the relationship between the axial distribution of pressure oscillation and the steam cavity, a method is proposed to determine the maximum steam cavity length by measuring the pressure oscillation distributions. The predicted deviation is only in range of $\pm 16 \%$ for the test conditions.

Keywords: Direct contact condensation; Flow pattern; Pressure oscillation; Steam cavity

\section{Introduction}

The steam jet condensation is a special form of steam-water direct contact condensation. It is widely used in industrial production, such as the surge tank and pressure suppression pool in nuclear reactor. The surge tank and pressure suppression pool plays an important role to ensure safety of nuclear reactor. When the primary circuit pressure of nuclear reactor exceeds the safety value that surge tank could maintain, steam will be jetted into the pressure suppression pool through spargers to ensure safety of nuclear reactor. Then the direct contact condensation occurs. Once the steam is jetted into water, the momentum and energy begin to exchange between steam and surrounding water drastically which affect the safety of equipment and nuclear reactor greatly. So the research on steam jet condensation is significant to the design and operation of safety system in nuclear reactor.

For the steam jet condensation, a lot of works have been developed for many years, like the condensation regime (Cho et al., 1998; Petrovic de With et al., 2007; Wu et al., 2009, Song and Kim, 2011), the steam jet pattern (Chun et al., 1996; Wu et al., 2009), the coefficient of heat transfer (Kim et al., 2001; Shah et al., 2010; Shah et al., 2013, Patel et al., 2014) and the steam jet condensation load (Fukuda, 1982; Simpson and Chan, 1982). When steam is jetted into water and condensed by water, there will generate some pressure impulse in water pool, which is called condensation oscillation. The pressure impulse may be harmful to the steam jet devices, such as the water pool. Especially, when the condensation oscillation frequency is close to the natural frequency of device, resonance will happen which may make great damage to the pool.

To avoid this damage, much attention has been paid to condensation oscillation for many years (Fukuda, 1982; Simpson and Chan, 1982, Nariai and Aya, 1986). In the past few years, many investigations have been focused on the low mass flux region, for example the chugging region(C), transient chugging region(TC) and condensation oscillation region (CO), as shown in figure 1. Fukuda (1982) investigated the shapes of steam cavity at low mass flux and found that the shapes of steam cavity correspond to the condensation oscillation. Also he found that the condensation oscillation frequency was proportional to the water subcooling and inverse 
proportional to the nozzle diameter. Chan and Lee (1982) investigated the flow and condensation pattern under low mass flux. Three typical condensation patterns were observed. They were the oscillation jet, chugging and bubble jet. Simpson and Chan (1982) made a further effort on low mass flux steam condensation. The process of bubble growth, bubble translation, and bubble separation (necking) was found and the process occurred periodically. Couple with the bubble separation process, pressure pulse was appeared periodically. Nariai and Aya (1986) researched the condensation oscillation frequency with three typical bubble shapes. They were the cylindrical bubble, spherical bubble and hemispherical bubble. The predicted frequency showed good corresponding to the experimental data. Aya et al. (1991) made some efforts on steam jets pressure oscillation in the chugging region and condensation oscillation region. Pressure oscillation was thought to be induced by the balance action between the supply of steam to steam cavity and its condensation on the steam-water interface. Tanskanen et al. (2014) simulated steam condensation for chugging regime, the information of bubble size and chugging frequency was revealed. Youn et al. (2003) found that when the steam bubble detached from the nozzle exit with the necking phenomenon, high pressure pulses with relatively low frequency were observed. To investigate the condensation oscillation frequency, the Strouhal number was adopted and it was related with Jacob number, Reynolds number, Weber number by previous researchers (Damasio et al., 1985; Simpson and Chan, 1982). Cho et al. (2004) investigated the characteristics of pressure oscillations in condensation oscillation (CO) region with multi-hole sparger and confirmed that the amplitude of the pressure pulse showed a peak at the pool temperature range of $45-85^{\circ} \mathrm{C}$. The dominant frequency was also affected by the pitch-to-hole diameter. Park et al. (2007) performed the tests in condensation oscillation (CO) and stable condensation (SC) region to a multi-hole sparger. At stable condensation (SC) region, the pressure oscillation for multi-hole was also found and it was different with the pressure oscillation of single hole.

\section{Figure 1 Condensation regime map (Cho et al., 1998)}

However, the research on the oscillation of steam jet condensation at stable condensation is relative less than the research on the low mass flux and oscillation condensation region. According to Hong et al. (2012), the steam condensation oscillation for stable condensation region (SC) was generated by the variation of steam jet length. Based on that, a one-dimensional oscillation model was proposed to predict the steam condensation oscillation frequency. Qiu et al. (2014a; 2014b; 2015) investigated the pressure oscillation with sonic and supersonic nozzles. Both the oscillation intension and dominant frequency was researched. Besides the first domain frequency, the second dominant frequency was found under some test condition.

Furthermore, although the steam cavity is thought to be related with the steam condensation oscillation, the relationship between steam cavity length and condensation oscillation at stable condensation region is rarely studied. Also, there are few works on the pressure oscillation intensity distribution along the axial direction. In order to solve these problems, the condensation oscillation and steam cavity for high mass flux is researched which is of signification to the industry application. With help of high speed camera and dynamic pressure sensors, the photos of steam cavity under high sample rate and pressure oscillation are acquired and analyzed, respectively. Also, the relationship between the steam jet pattern and pressure oscillation is investigated. 


\section{Experimental apparatus and measurement point}

\subsection{Experimental apparatus}

The experimental apparatus of steam jet submerged in quiescent water adopts the system which was reported by Qiu et al. (2014a), as shown in figure 2. Steam is generated at steam generator and then flow through the steam pipe. Finally, it is jetted into subcooled water with a nozzle and then condensed. Steam mass flux is controlled by an electric control valve and measured by vortex type steam flow meter which accuracy is $0.5 \%$ full span(FS). The steam pressure and temperature before nozzle inlet are measured with a pressure transducer $(0.1 \%$ full span) and a T-type thermocouple $\left(0.5^{\circ} \mathrm{C}\right)$, respectively. Eight T-type thermocouples are distributed uniformly to measure the pool water temperature. The test nozzles are installed horizontally and submerged with depth of $500 \mathrm{~mm}$ in square tank. The nozzles with inner diameters of $8 \mathrm{~mm}$ and $10 \mathrm{~mm}$ are tested.

Two high frequency dynamic pressure sensors are used to measure the pressure oscillation, and they are equipped on the three-dimensional mobile mounting bracket. The dynamic pressure sensors are manufactured by the Xi'an Jiecheng sensor measurement and control technology company limited and the model is CYG41000T. Also, the pressure sensors have been calibrated by the Changcheng institute of metrology \& measurement which has the certificates of lab accreditation issued by China national accreditation board for laboratories. The resonant frequency of dynamic pressure sensors is $26 \mathrm{kHz}$ and it can acquire the dynamic pressure signal with accuracy of $0.5 \%$ full span (FS). Then the pressure signal is transformed and acquired by a dynamic pressure acquisition cards (NI 4472) and transimitted to the industrial personal computer. The noise level is tested, and the maximum noise level is turned out to be about $0.01 \mathrm{kPa}$. A high speed camera (Phantom V611) is applied to take photos of steam cavity. The maximum photo sample rate can reach to 100000 frames per seconds.

\section{Figure 2 Schematic diagram of experimental system(Qiu et al., 2014a)}

\subsection{Measurement point}

The test parameters are shown in table 1. Two high frequency dynamic pressure sensors are installed at the three-dimension mobile scaffold, as shown in figure 3. Sensor probes and nozzle centre line are in the same horizontal plane, and sensor probes are vertical to the steam jet direction. The position of dynamic pressure sensors could be adjusted by the three-dimension mobile scaffold. The horizontal plane where the nozzle centre line locates is adopted as the reference plane and nozzle exit centre is used as the origin of coordinates. The steam jet direction is defined as the $\mathrm{X}$ axis and the nozzle radial direction is difined as the $\mathrm{R}$ axis. Also, the dimensionless axis distance $\mathrm{X} / \mathrm{D}$ and radial distance as R/D is difined, respectively, where the $\mathrm{D}$ is the diameter of nozzle. At initial moment, two sensors are fixed at the position of $\mathrm{X} / \mathrm{D}=0,10$ respectively, with $R / D=2$. Then the sensors are moved along the $X$ axis simultaneously. The moving distance is $\Delta \mathrm{X} / \mathrm{D}=1$ for every step, as show in figure 4 . 
Figure 3 Dynamic pressure sensors and fixed bracket

Figure 4 Distribution of measurement points

\subsection{Experimental method and repeats}

To compare the condensation oscillation for different test condition, the root mean square value of pressure oscillation which can reflect the average oscillation intension is adopted to estimate the pressure oscillation intension.

Mean pressure oscillation intension:

$$
P=\sqrt{\frac{\sum_{i=1}^{N}\left(P_{i}-\bar{P}\right)^{2}}{N}}
$$

Where $P_{i}$ is the instantaneous pressure and $N$ is the sample number. $\bar{P}$ is the mean pressure and it can be expressed as follow.

$$
\bar{P}=\frac{1}{N} \sum_{i=1}^{N} P_{i}
$$

The experimental repeatability is also tested. The experiment for the condition of water subcooling $\Delta \mathrm{T}=45^{\circ} \mathrm{C}$ and mass flux $\mathrm{G}=500 \mathrm{~kg} \cdot \mathrm{m}^{-2} \cdot \mathrm{s}^{-1}$ are conducted twice. Figure 5 shows the results of the two experiments. It indicates that the experimental results have a good reproducibility.

Figure 5 Repeatability of experiment

\section{Experimental results}

\subsection{Flow pattern and pressure oscillation}

According to previous researches, the pressure oscillation is closely related to the flow patterns. Although previous have reported that the steam cavities at stable condensation region (SC) are still unstable, and the pressure oscillation is also measured in this condensation region, steam cavity shapes are quite different under different photo sample rate. Under low photo sample rate, the edge of steam cavities is smooth and the shapes are similar to that observed by eyes ( 24 frames per seconds) directly, as shown in Figure 6. Additionally, small variation of steam cavity length is observed, even the sample rate reaches to 1000 frames per seconds, which may lead the measurement value of steam cavity length deviating from the real length.

But, when the photo sample rate increases with 5000 frames per seconds, clearly, the interface of steam cavity becomes rough and there are obvious changes for the steam cavity length. Also, some details of steam cavity are captured, such as the separated steam bubble, as shown in figure 6 .

Figure 6 Comparison of steam cavity shape between different photo sample rates

Figure 7 shows the variation of steam cavities which is obtained under photo sample rate of 5000 frames per seconds. The interval time for two continuous photos is 0.0002 seconds. As a reference, a line is put at the steam cavity tail which represents the average length of steam cavity 
in a cycle, approximately. Obviously, the steam cavity varies all the time and the cavity lengths prolong and shorten periodically. The deviation between the maximum and minimum steam jet length is even up to $30 \%$ of average steam jet length. Due to the tremendous changes of steam cavity length, the pressure oscillation occurs. Moreover, the variation of steam cavity length would make it hard to measure the steam cavity length, and the measurement result may produce significant errors. Thus, in this paper a method based on the pressure oscillation is used to determine the maximum steam cavity length.

Furthermore, from the figure 6 and figure 7, the separated bubbles are found at the end of steam cavity which is marked with circle line. In the process of steam cavity length variation, the length of steam cavity prolongs gradually at first, and then an obvious necking appears when the steam cavity approximates to the maximum. With the further development of steam cavity length, a steam bubble separates from the steam cavity and the steam cavity becomes short again. With the variation of steam cavity, the phenomena of steam cavity necking, and bubble separation would emerge continuously at the end of steam cavity. Then, under the condensation process and force of steam bubble inner pressure, water pressure and the bubble surface tension, the separated bubble oscillates and the pressure pulse generates periodically.

\section{Figure 7 Continuous photos of steam cavity}

Coupled with the circulation of steam cavity shape variation and bubble separation, the pressure oscillation wave appears. Figure 8 shows the pressure oscillation wave in a period of time. Also, when the pressure oscillation signal is transformed by the Fast Flourier Transform (FFT), the oscillation frequency is obtained, as shown in figure 9. In the frequency spectrum, two obvious dominant frequencies appear and they are named as the first dominant frequency and the second dominant frequency, respectively. According to the report of Hong et al.(2012), the first dominant frequency is caused by the variation of steam cavity length and the theoretic oscillation frequency for the variation of steam cavity length is obtained which well matches the first dominant frequency obtained from the experiment. Qiu et al.(2015) also proved that the first dominant frequency is generated by the variation of steam cavity length with the sonic and supersonic nozzles.

For the second domain frequency, Qiu et al.(2014b) have demonstrated that it is generated by the steam bubble through the analysis on the steam bubble photos. Additionally, with measuring the steam bubble diameter from the photo, the steam bubble oscillation frequency is estimated by using the bubble oscillation characteristic frequency equation which is derived by Leighton (1994) and Song et al. (2011) based on the Rayleigh equation. It is found that the steam bubble oscillation frequency conforms to the second dominant frequency in frequency spectrum. So, it can be deduced that the second dominant frequency is generated by the steam bubble. In conclusion, it indicates that the complicated condensation pressure oscillation signal is not only caused by the variation of steam cavity length, but also caused by the steam bubble oscillation. It is the joint action of steam cavity length variation and steam bubble oscillation. Also, clearly, the frequency caused by bubble oscillation is higher than the frequency generated by steam jet length variation. 


\subsection{Axial distribution of pressure oscillation intension}

Comparing the pressure oscillation intension for different point, it is found that at position of $\mathrm{X} / \mathrm{D}=5$ and $\mathrm{X} / \mathrm{D}=10$, the amplitude of pressure oscillation waves is very high, however, for other measurement point, such as the position near the nozzle outlet, the pressure oscillation amplitudes are very small, as shown in figure 10. Generally, the amplitude of oscillation pressure impulse increased firstly and then decreased with the increase of X/D.

\section{Figure 10 Pressure oscillation for different measurement point}

To evaluate the average intension of pressure oscillation for different measurement point, the dynamic pressure data shown in figure 10 is dealt with Eq.(1), and the mean pressure oscillation intension is adopted to evaluate the level of condensation oscillation. Thus, the axial distribution of pressure oscillation intension is obtained and compared. Figure 11 shows the axial pressure oscillation intension distributions under the test condition. Similar with the tendency of original pressure pulse, the pressure oscillation intension increases firstly and then decays with going far away from nozzle exit. There existed an obvious pressure oscillation peak at some measurement point.

Furthermore, with the rise of steam mass flux, the steam energy increases, then the oscillation energy will increase which leads the pressure oscillation intension becoming stronger. Also, with increasing of steam mass flux, the interface area between the steam and water increases and the pressure oscillation intension increases rapidly. However, when water temperature increases, the pressure intension decreases firstly, and then increases gradually.

Figure 11 (a)

Figure 11(b)

Figure 11 Axial pressure oscillation intension distributions verse the pool water temperature and steam mass flux

The distribution characteristic of pressure oscillation intension can be analyzed with steam flow pattern. According to the analysis on the steam jet pattern and pressure oscillation wave, it could deduce that the pressure oscillation is caused by the joint action of steam cavity length variation and steam bubble oscillation. At the process of steam condensation, the length of steam cavity changes, then the space at the end of steam cavity will occupied by the steam and water alternatively. Also, according to the polytrophic process equation, the pressure at the end of steam cavity will change when the steam cavity length varies. Thus, the pressure oscillation occurs. Moreover, according to the report of Leighton (1994) and Song et al.(2011), under the function of force, bubble will oscillate and cause pressure oscillation periodically. And a single steam bubble condensation behaviors in subcooled water have been simulated using Moving Particle Semi-implicit (MPS) method by Tian et al.,(2010). It was found that the both the size of steam bubble and its inner pressure changed violently which would cause the pressure oscillation in water. Also, the second dominant frequency in figure 9 has been proved to be generated by the separated steam bubble. And the amplitude of the second dominant is bigger than that of the first dominant frequency near the end of steam cavity. Thus, the pressure oscillation peak is also the 
superposed influence of steam cavity length variation and bubble oscillation. And the bubble oscillation even acts the major role in the formation of the pressure oscillation intension peak.

Moreover, figure 7 shows that both the axial positions where steam cavity lengths vary and steam bubbles separate locate at the end of steam cavity. According to wave theory, there must be a pressure oscillation intension peak appears at this position. When oscillation wave propagates away from the pressure oscillation intension peak, it would be damped by water gradually. Then, the oscillation intension decreases gradually with the measurement point moving away from condensation oscillation peak, as shown in figure 11.

\section{3 Relationship between the steam cavity and pressure oscillation axial distribution}

According to the analysis, conclusions have been obtained that the pressure oscillation is caused by the steam cavity length variation and steam bubble oscillation. The pressure oscillation peak is the combined effect of the steam cavity length variation and steam bubble oscillation. Furthermore, the position of pressure oscillation peak is deduced to be at the end of steam cavity. Then the pressure oscillation peak position is compared with the steam cavity, as shown in figure 12. It shows that the pressure oscillation peak is well corresponding to the end of the maximum steam cavity in a cycle.

$$
\text { Figure 12(a) }
$$

Figure 12 (b)

Figure 12 Corresponding relation of axial pressure oscillation intension distributions and steam cavity shapes

Although both of the two oscillation modes happen at the steam cavity tail, further analysis shows that there are some differences between them. According to the steam cavity length oscillation model at stable condensation region (Hong et al., 2012), condensation oscillation equilibrium position is the average length of steam cavity. But the position where the steam bubble oscillates is different with the steam cavity length oscillation. As shown in figure 7, it clearly shows that there is no bubble separating from the steam cavity before the steam cavity prolongs to the maximum. The steam bubbles begin to separate only at the time when the steam jet length prolongs to its maximum, approximately. Then the separated steam bubbles oscillate and the second dominant frequency is generated. Moreover, based on the frequency spectrum shown in figure 9, the second dominant frequency band generated by the separated bubble is a major element in the frequency spectrum. And the oscillation amplitude of the second dominant frequency is even bigger than the amplitude of the first dominant frequency at the region near the end of steam cavity. All these phenomena indicate that the separated steam bubble oscillation has important impact on the pressure oscillation. Thus, based on the wave theory, it is reasonable that an oscillation peak occurs at the end of maximum steam cavity length where the steam bubble separates.

\subsection{Energy analysis for condensation oscillation}

Further to certify the influence of the separated steam bubble condensation and oscillation process on the pressure oscillation, the energy generated by the bubble separation and condensation is analyzed based on the frequency spectrum. Firstly, the second dominant frequency is the pressure oscillation frequency of bubble oscillation, so the energy of the second dominant 
frequency band can reflect the energy generated by the separated steam bubble oscillation. As a comparison, the energy generated by the variation of steam cavity length is also studied and its energy can be reflected from the energy of the first dominant frequency band.

The energy of each dominant frequency band can be obtained from the frequency spectrum with the Parseval's relation (Smith, 2003).

$$
E=\int_{t_{1}}^{t_{2}}|f(t)|^{2} \mathrm{~d} t=\frac{1}{2 \pi} \int_{\omega_{1}}^{\omega_{2}}|F(j \omega)|^{2} \mathrm{~d} \omega
$$

Where the $f(t)$ is the pressure oscillation signal in time spectrum, the $t_{1}$ and $t_{2}$ is the time region. $F(\omega)$ is the pressure signal in frequency spectrum which is transformed with the method of Fast Fourier transform(FFT). $\omega_{1}$ and $\omega_{2}$ stand for the frequency region.

Figure 13 shows the axial distribution of energy for the first dominant frequency band and the second dominant frequency band, respectively. With the axial position being away from the nozzle outlet, the energy of the second dominant frequency band increases at first and then deceases. There is also an obvious energy peak at the end of steam cavity. Similarly, it is undoubted that the bubble separation, oscillation and condensation occur at the steam tail. However, the energy of the first frequency band is small when compared with that of the second dominant frequency band. And with the measurement position moving to downstream, the energy of the first frequency band changes little.

\section{Figure 13(a) Figure 13(b) \\ Figure 13 Energy distributions along the axial position}

Figure 14 shows the comparison of the energy of these two frequency band. Although the oscillation energy of the second dominant frequency band is less than that of the first dominant frequency band at the first few measurement points, it increases rapidly and then become larger than that of the first dominant frequency band. Near the energy peak of the second dominant frequency band, the energy of the second dominant frequency band is much stronger than the energy of the first frequency band. It clearly demonstrates that the pressure oscillation intension caused by steam bubble oscillation is much stronger than that caused by the variation of steam cavity length. Additionally, it can be revealed that, near the end of steam cavity, the pressure oscillation is mainly contributed by the steam bubble oscillation. Thus, to avoid the damage of condensation oscillation, evading the steam bubble oscillation is very important.

\section{Figure 14(a) Figure 14(b) \\ Figure 14 Energy distributions for each frequency band}

When oscillation wave propagates away from the end of steam cavity, the energy will be dissipated by water gradually, so the oscillation energy decreases slowly. Furthermore, according to the wave theory, the wave energy decay rate is affected by the wave frequency. As higher is the oscillation frequency, the faster the energy is attenuation. Thus, it is reasonable that the decay rate for energy generated by steam bubble oscillation is faster than that of energy generated by steam cavity variation because the steam bubble oscillation frequency is higher than steam cavity variation frequency. Moreover, the energy of the second dominant frequency band is less than that 
of the first dominant frequency band is understandable when the measurement points are far away from the steam cavity tail.

\subsection{Dimensionless position of oscillation intension peaks}

Figure 15 shows the axial position of pressure oscillation peak changes with the variation of steam mass flux and pool water temperature. Generally, the peak position moves towards downstream gradually with the rise of pool water temperature and steam mass flux. Due to the conclusion that the pressure oscillation peak is the combined effect of the steam cavity length variation and steam bubble oscillation, thus, when pool water temperature increases, the water condensing capacity decreases. In order to condense all the steam provided, the steam cavity prolongs to increase heat transfer area. Thus, the position where steam bubble separates will move to downstream simultaneous with the rise of steam cavity length. As a result, the condensation oscillation peak moves to downstream naturally. Similarly, as the steam mass flux increases, the power steam brings increases and the steam jet length prolongs, then the oscillation peak moves back.

Figure 15 Dimensionless position of pressure oscillation intension peak

For the steam jet length, almost all the researchers gave a semi-empirical correlation to predict the dimensionless penetration length based on basic expression (Kerney et al., 1972).

$$
\frac{L}{D}=0.5\left(B S_{m}\right)^{-1}\left(\frac{G_{0}}{G_{m}}\right)^{0.5}
$$

Where $G_{m}$ is the average steam mass flux, $S_{m}$, expressed as $h / C p \cdot G_{0}$, is the mean transport modulus and $\mathrm{B}$ represents the water condensation capacity.

According to figure 15, the dimensionless axial position where the pressure oscillates strongest is also mainly affected by the steam mass flux and water temperature, and it increases with steam mass flux rise, but decreases with rise of water condensation capacity, which is very similar to the characteristic of steam jet length. Thus, based on the original expression given by Kerney et al.(1972), a correlation is proposed to predict the dimensionless axial peak position, as shown in Eq.(5). Almost all of the experimental data lied within the range of $\pm 10 \%$, as shown in figure 16. Thus, with Eq.(5), the axial position where pressure oscillates strongest can be obtained and it is significant for industry application to prevent the pressure oscillation.

$$
\frac{L}{D}=0.663 B^{-0.55}\left(\frac{G_{0}}{G_{\mathrm{m}}}\right)^{0.93}
$$

Figure 16 Deviations of dimensionless axial oscillation peak position based on Eq.(5)

Since the strongest pressure oscillation occurs at the end of steam cavity and the distance from pressure oscillation peak positions to nozzle outlet corresponds well with the maximum steam jet lengths, so the maximum steam cavity length can be obtained by measuring the pressure oscillation intension along with the axial direction of steam cavity and finding out the point where the pressure oscillate is strongest, then the distance from the strongest oscillation point to the nozzle outlet is the maximum length of steam cavity length. Moreover, the Eq.(5) which is used to 
predict the pressure oscillation peak position also can be applied to predict the maximum steam cavity length.

\subsection{Maximum steam cavity length}

For the design of submerged gas jet equipments, another principal requirement is the information of the jet cavity shape and its dimension. It is closely related with steam cavity shape, heat transfer and condensation oscillation. Generally, combining the steam cavity length with the distribution of steam axial temperature, the coefficient of heat transfer can be obtained. Also the condensation oscillation frequency is also affected by the steam cavity length (Hong et al., 2012). For the steam jet pump, the maximum steam cavity length is the basis for the design of mixing section length. Reasonable design for mixing section length will raise the efficiency of steam jet pump greatly (Shah et al., 2011; Shah et al., 2013). Thus, the research on the steam jet length is very important for the industry.

At present, the steam jet length is mainly obtained by measuring from the steam cavity photos (Kerney et al., 1972; Weimer et al., 1973; Chun et al., 1996; Park et al., 1999; Kim et al., 2001) or from the axial temperature distribution (A Kudo et al., 1974, Del Tin et al., 1983). However, the accuracy of steam cavity lengths with these two methods is poor. The accuracy of jet length measured from steam cavity photograph is determined by the spatial resolution of taking pictures, the light condition in water and image analysis. For the method based on the axial temperature distribution along steam central line, the flow field is easy to be disturbed by the temperature measuring equipment, and then the end of steam cavity is hard to distinguish. The most important of all, when the steam cavities are unstable and varied rapidly, the precision of measurement result may be very poor. Certainly, the accurate of steam cavity lengths is dubious.

Based on the character that condensation oscillation intension peak located at the end of maximum steam cavity, the oscillation intension peak position also can be applied to predict the maximum steam cavity length. Finding out the point where the pressure oscillates strongest and measuring the distance from the strongest oscillation point to the nozzle outlet, then the maximum value of steam cavity length can be obtained. When compared with traditional methods, it has many advantages over the traditional method. Firstly, it is not affected by the limitation of light and photo equipment and there is little effect on the flow field. The most advantage characteristic is that it can make full use of the vibration of steam cavities which is relatively accurate for the prediction of steam cavity length.

To test this method, the steam cavity shape is also obtained with high speed camera. Figure 17 shows the maximum dimensionless jet lengths measured from the photos under different steam mass flux and water temperature. It indicates that the maximum steam dimensionless jet length prolongs gradually with the rise of pool water temperature and steam mass flux, which is similar to the tendency of the average steam dimensionless jet length by previous (Kerney et al., 1972; Chun et al., 1996; Kim et al., 2001). Additionally, the tendency and values of maximum dimensionless steam cavity lengths are almost the same to that of the pressure oscillation peak position.

Figure 17 Maximum dimensionless steam jet lengths under different steam mass flux and water temperature 
Figure 18 shows the comparison of predicted steam cavity length by Eq.(5) with experimental data for all the test conditions. The deviation of prediction is only in range of $\pm 16 \%$. It indicates that the length predicted by Eq.(5) corresponds to the maximum steam cavity length very well. So, the method by measuring the condensation oscillation peak position to identify the maximum steam jet length is very accurate, also it is convenient and utility for some conditions that other methods are limited.

Figure 18 Deviations of the predicted maximum steam cavity length based on Eq.(5)

\section{Conclusions}

Submerged steam jet condensation is widely applied in industrial applications, and the research on steam jet condensation oscillation is significant for the industrial equipments. However, the research on the pressure oscillation at stable region is relatively less. In this paper, combining with the steam jet pattern at stable condensation region, the pressure oscillation intension and its axial distribution is investigated clearly. Moreover, the relationships between them are also researched. The main conclusion can be summarized as follows.

(1) At SC region, steam jet cavity shape is unsteady. With the variation of steam cavity length and bubble separation, the pressure oscillation occurs.

(2) Along the steam cavity axial direction, there is an obvious pressure oscillation peak and the axial position of pressure oscillation peak well corresponds to the maximum steam cavity length. Coupled with the analysis on the steam cavity pattern, it shows that the pressure oscillation peak is the combined effect of steam bubble oscillation and variation of steam cavity length.

(3) Energy analysis shows that the energy generated by the steam bubble is larger than the energy generated by steam cavity length variation and the steam bubble oscillation plays a dominant role in condensation oscillation. Thus, it is reasonable that the oscillation peak well matches the maximum steam cavity length.

(4) The dimensionless length from the oscillation peak position to nozzle outlet increases with steam mass flux rise and decreases with the rise of water subcooling degree which is similar to that of steam cavity length. Based on the relationship between condensation oscillation distribution and the steam flow pattern, maximum steam cavity length can be judged by measuring the oscillation peak position. The predicted deviation is only in range of $\pm 16 \%$.

\section{Acknowledgements:}

This work was supported by the national science foundation of china (No. 51476128, and No.51125027), the national key basic research development plan (No.2015CB251504).

\section{Figure Captions}

Figure 1 Condensation regime map

Figure 2 Schematic diagram of experimental system(Qiu et al., 2014a)

Figure 3 Dynamic pressure sensors and fixed bracket

Figure 4 Distribution of measurement point

Figure 5 Repeatability of experiment

Figure 6 Comparison of steam cavity shape between different photo sample rates 
Figure 7 Continuous photos of steam cavity

Figure 8 Pressure oscillation wave in a period of time

Figure 9 Frequency spectrum of condensation oscillation

Figure 10 Pressure oscillation for different measurement point

Figure 11 Axial pressure oscillation intension distributions verse the water temperature and steam mass flux

Figure 12 Corresponding relation of axial pressure oscillation intension distributions and steam cavity shapes

Figure 13 Energy distributions along the axial position

Figure 14 Comparison of the energy in each frequency band

Figure 15 Dimensionless position of pressure oscillation intension peak

Figure 16 Deviations of dimensionless axial oscillation peak position based on Eq.(5)

Figure 17 Maximum dimensionless steam jet lengths under different steam mass flux and water subcooling

Figure 18 Deviations of the predicted maximum steam cavity length based on Eq.(5)

Table Captions

Table 1 Test conditions in experiment

\section{References:}

A Kudo, T Egusa S, Toda, 1974. Basic study of vapor suppression. In Proceedings of the 5th International Heat Transfer Conference, Tokyo, 221-225.

Aya, I. ,Nariai, H., 1991. Evaluation of heat-transfer coefficient at direct-contact condensation of cold water and steam. Nuclear Engineering and Design. 131, 17-24.

Chan, C. K. ,Lee, C., 1982. A regime map for direct contact condensation. International Journal of Multiphase Flow. 8, 11-20.

Chan, C. K., 1978. Dynamical pressure pulse in steam jet condensation. In Proc. 6th Int. Heat Transfer Conf. Toronto, 395-399.

Cho, S., Song, C. H., Park, C. K., Yang, S. K. ,Chung, M. K., 1998. Experimental study on dynamic pressure pulse in direct contact condensation of steam discharging into subcooled water. In The 1st Korea-Japan Symposium on Nuclear Thermal Hydraulics and Safety, Pusan, Korea, 291-298.

Cho, S., Chun, S., Baek, W. ,Kim, Y., 2004. Effect of multiple holes on the performance of sparger during direct contact condensation of steam. Experimental Thermal and Fluid Science. 28, 629-638.

Chun, M., Kim, Y. ,Park, J., 1996. An investigation of direct condensation of steam jet in subcooled water. International Communications In Heat and Mass Transfer. 23, 947-958.

Del Tin, G., Lavagno, E. ,Malandrone, M., 1983. Thermal and fluid-dynamic features of vapor condensing jets. Heat Tech. 1, 13-35.

Fukuda, S., 1982. Pressure variations due to vapor condensation in liquid (II): phenomena at large vapor mass flow rate, J. Atom. Energy Soc. Japan 24 (1982) 466-474.

Hideki Nariai, I. A., 1986. Fluid and pressure oscillations occuring at direct contact condensation of steam flow with cold water. Nuclear Engineering and Design. 95, 35-45.

Hong, S. J., Park, G. C., Cho, S. ,Song, C., 2012. Condensation dynamics of submerged steam jet 
in subcooled water. International Journal Of Multiphase Flow. 39, 66-77.

Kerney, P. J., Faeth, G. M. ,Olson, D. R., 1972. Penetration characteristics of a submerged steam jet. Aiche Journal. 18, 548-553.

Kim, H. Y., Bae, Y. Y., Song, C. H., Park, J. K. ,Choi, S. M., 2001. Experimental study on stable steam condensation in a quenching tank. International Journal of Energy Research. 25, 239-252.

Leighton T. 1994, the Acoustic Bubble. Academic press. pp:130-139.

Nariai, H., Aya, I., 1986. Fluid and pressure oscillations occuring at direct contact condensation of steam flow with cold water. Nuclear Engineering and Design. 95, 35-45.

Park, C. K., Song, C. H. Jun, H. G., 2007. Experimental investigation of the steam condensation phenomena due to a multi-hole sparger. Journal of Nuclear Science and Technology. 44, 548-557.

Park, C. K., Cho, S., Choi, N. H., Yang, S. K. ,Chung, M. K., 1999. A study on the characteristics of the jet type condensation. In 99 Korea Nuclear Society Spring Conference.

Patel, G., Tanskanen, V. , Kyrki-Rajamäki, R., 2014. Numerical modelling of low-Reynolds number direct contact condensation in a suppression pool test facility. Annals of Nuclear Energy. 71, 376-387.

Petrovic de With, A., Calay, R. K. ,de With, G., 2007. Three-dimensional condensation regime diagram for direct contact condensation of steam injected into water. International Journal of Heat And Mass Transfer. 50, 1762-1770.

Qiu, B., Tang, S., Yan, J., Liu, J., Chong, D. ,Wu, X., 2014a. Experimental investigation on pressure oscillations caused by direct contact condensation of sonic steam jet. Experimental Thermal and Fluid Science. 52, 270-277.

Qiu, B., Yan, J., Liu, J., Chong, D., Zhao, Q. ,Wu, X., 2014b. Experimental investigation on the second dominant frequency of pressure oscillation for sonic steam jet in subcooled water. Experimental Thermal and Fluid Science. 58, 131-138.

Qiu, B., Yan, J., Liu, J. ,Chong, D., 2015. Experimental investigation on pressure oscillation frequency for submerged sonic/supersonic steam jet. Annals of Nuclear Energy. 75, 388-394.

Shah, A., Chughtai, I. R. ,Inayat, M. H., 2010. Numerical simulation of direct-contact condensation from a supersonic steam jet in subcooled water. Chinese Journal of Chemical Engineering. 18, 577-587.

Shah, A., Chughtai, I. R. ,Inayat, M. H., 2011. Experimental and numerical analysis of steam jet pump. International Journal Of Multiphase Flow. 37, 1305-1314.

Shah, A., Khan, A. H., Chughtai, I. R. ,Inayat, M. H., 2013. Numerical and experimental study of steam-water two-phase flow through steam jet pump. Asia-Pacific Journal of Chemical Engineering. 8, 895-905.

Simpson, M. E. ,Chan, C. K., 1982. Hydrodynamics of a subsonic vapor jet in subcooled liquid. Journal of Heat Transfer. 104, 271-278.

Smith, S. W.,2003. Chapter 10 - Fourier Transform Properties. In Digital Signal Processing (First), S. W. Smith, ed.eds. (Boston, Newnes), pp. 185-208.

Song, C. ,Kim, Y., 2011. Direct Contact Condensation of Steam Jet in a Pool. Advances in Heat Transfer. 43, 227.

Tanskanen, V., Jordan, A., Puustinen, M. ,Kyrki-Rajamäki, R., 2014. CFD simulation and pattern recognition analysis of the chugging condensation regime. Annals of Nuclear Energy. 66, 
133-143.

Tian, WX., Ishiwatari, Y., Ikejiri, S., Yamakawa, M. ,Oka, Y., 2010. Numerical computation of thermally controlled steam bubble condensation using Moving Particle Semi-implicit (MPS) method. Annals Of Nuclear Energy. 37, 5-15.

Weimer, J. C., Faeth, G. M. ,Olson, D. R., 1973. Penetration of vapor jets submerged in subcooled liquids. Aiche Journal. 19, 552-558.

Wu, X., Yan, J., Pan, D., Liu, G. ,Li, W., 2009. Condensation regime diagram for supersonic/sonic steam jet in subcooled water. Nuclear Engineering and Design. 239, 3142-3150.

W.V. Pinczewski, 1981. The formation and growth of bubbles at a submerged orifice. Chemical Engineering Science, 36(2), 405-411.

Youn, D. H., Ko, K. B., Lee, Y. Y., Kim, M. H., Bae, Y. Y. ,Park, J. K., 2003. The direct contact condensation of steam in a pool at low mass flux. Journal of Nuclear Science And Technology. 40, 881-885. 


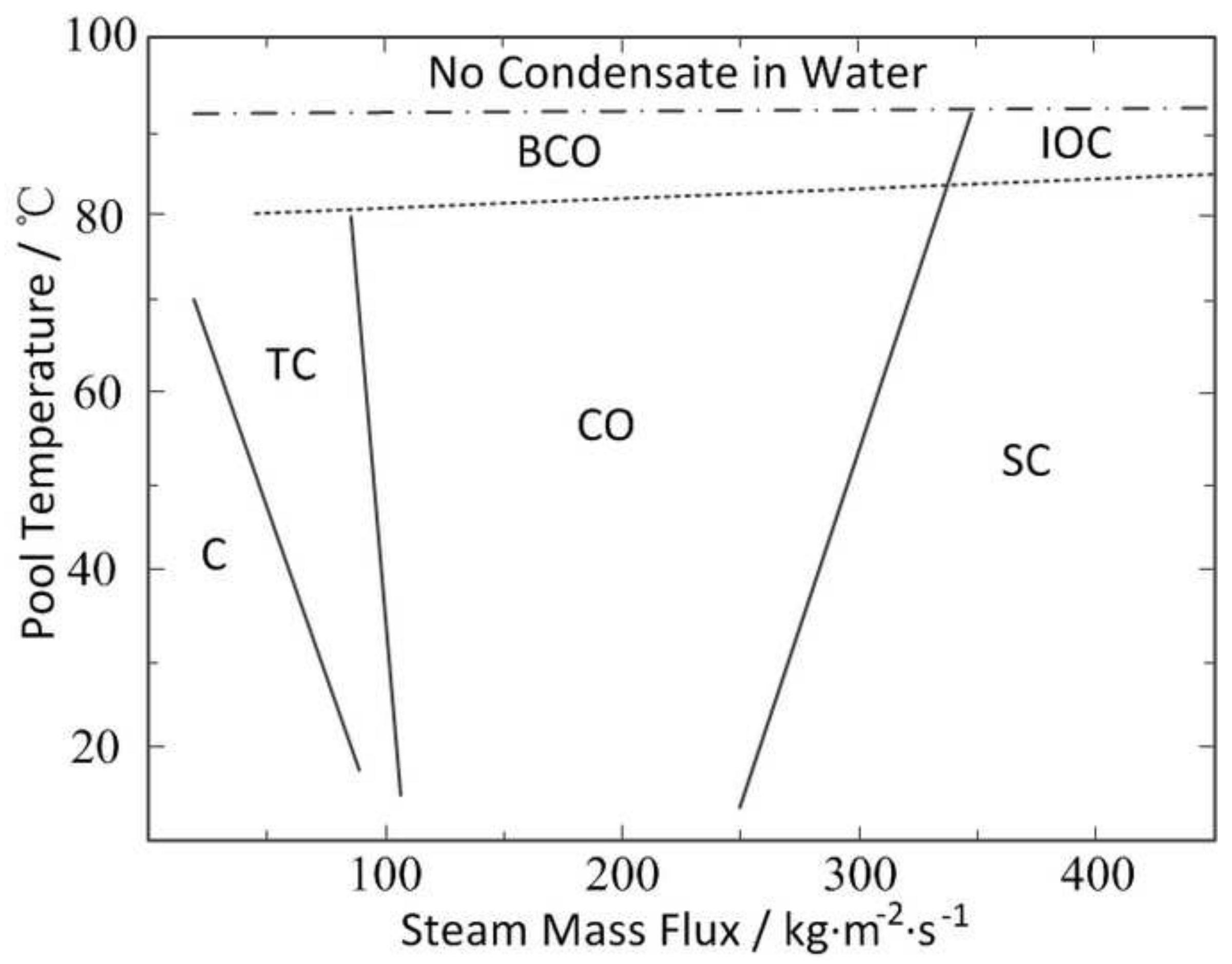




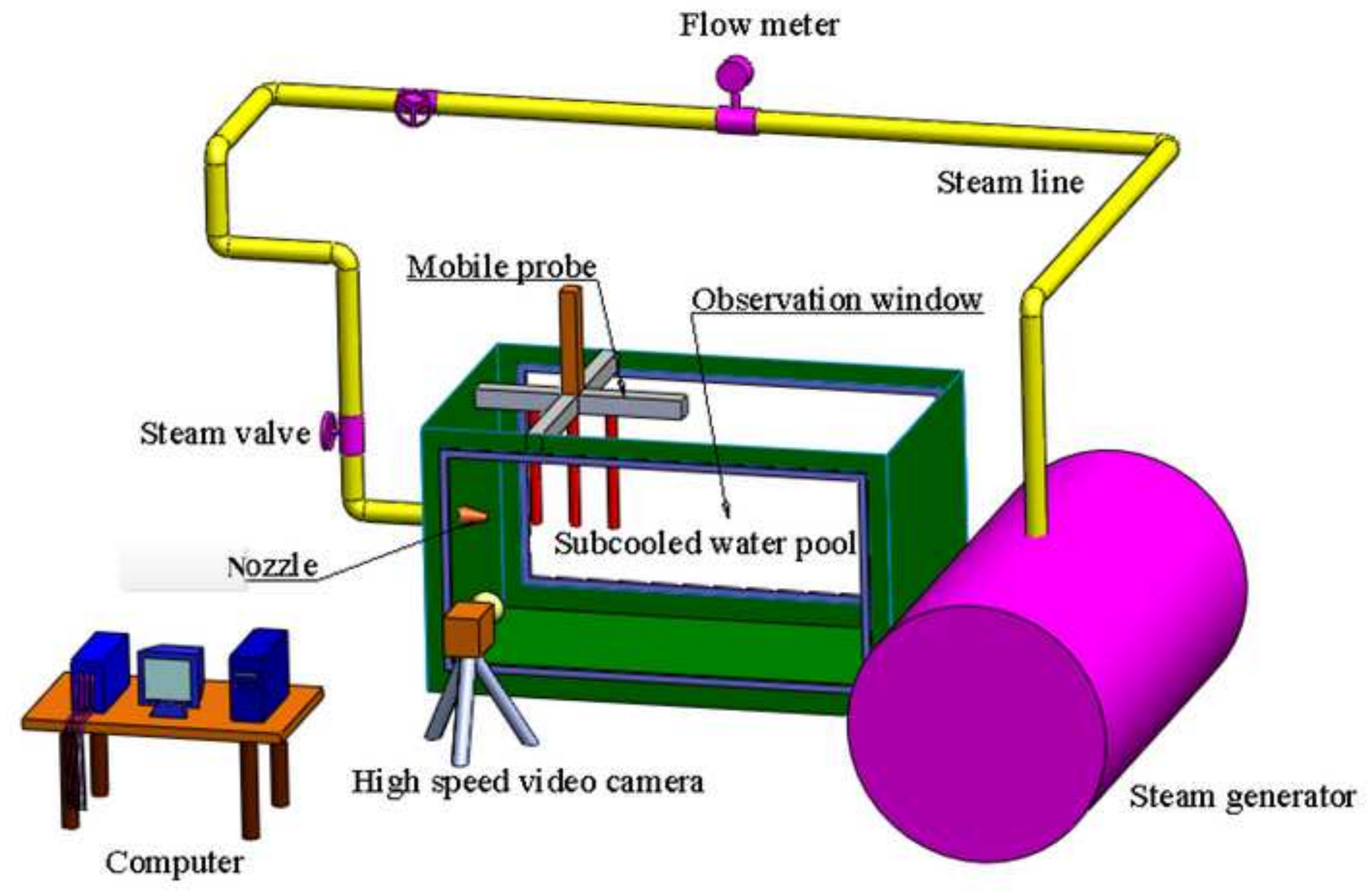




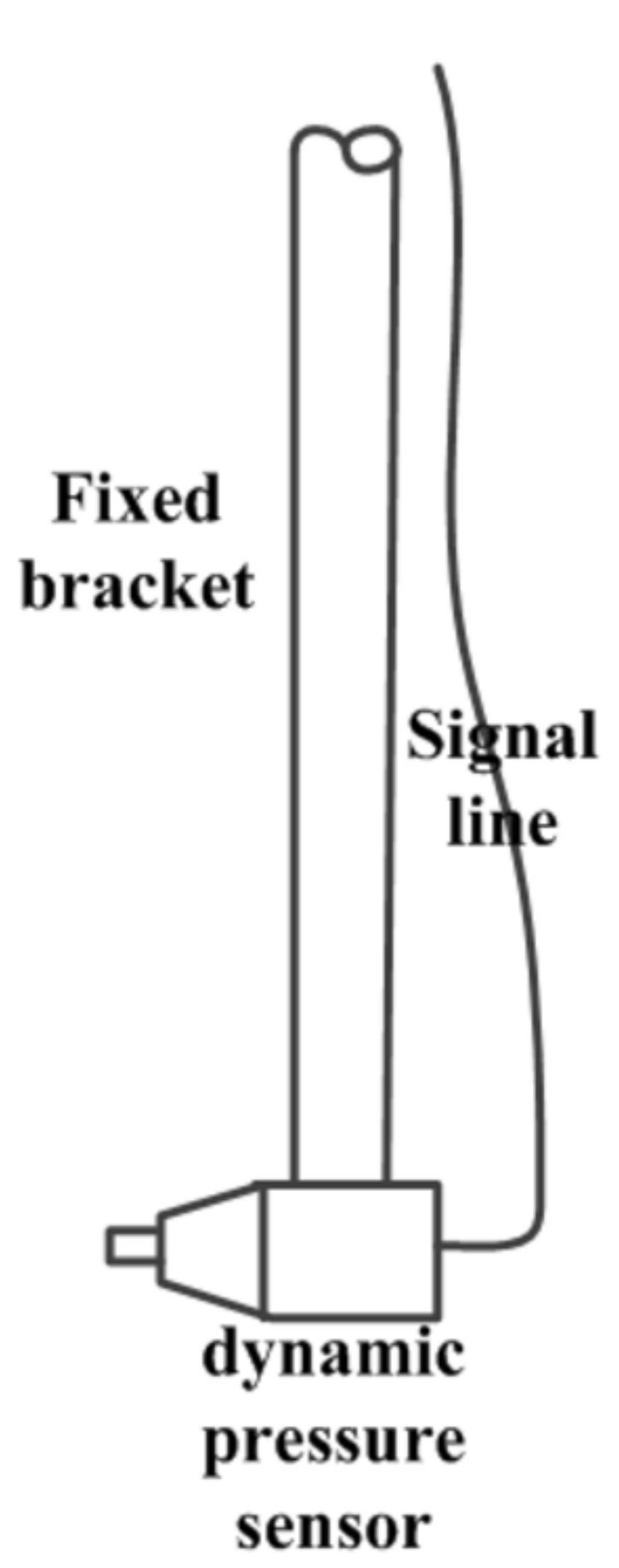

Fig.3

\section{Fixed bracket} sensor 


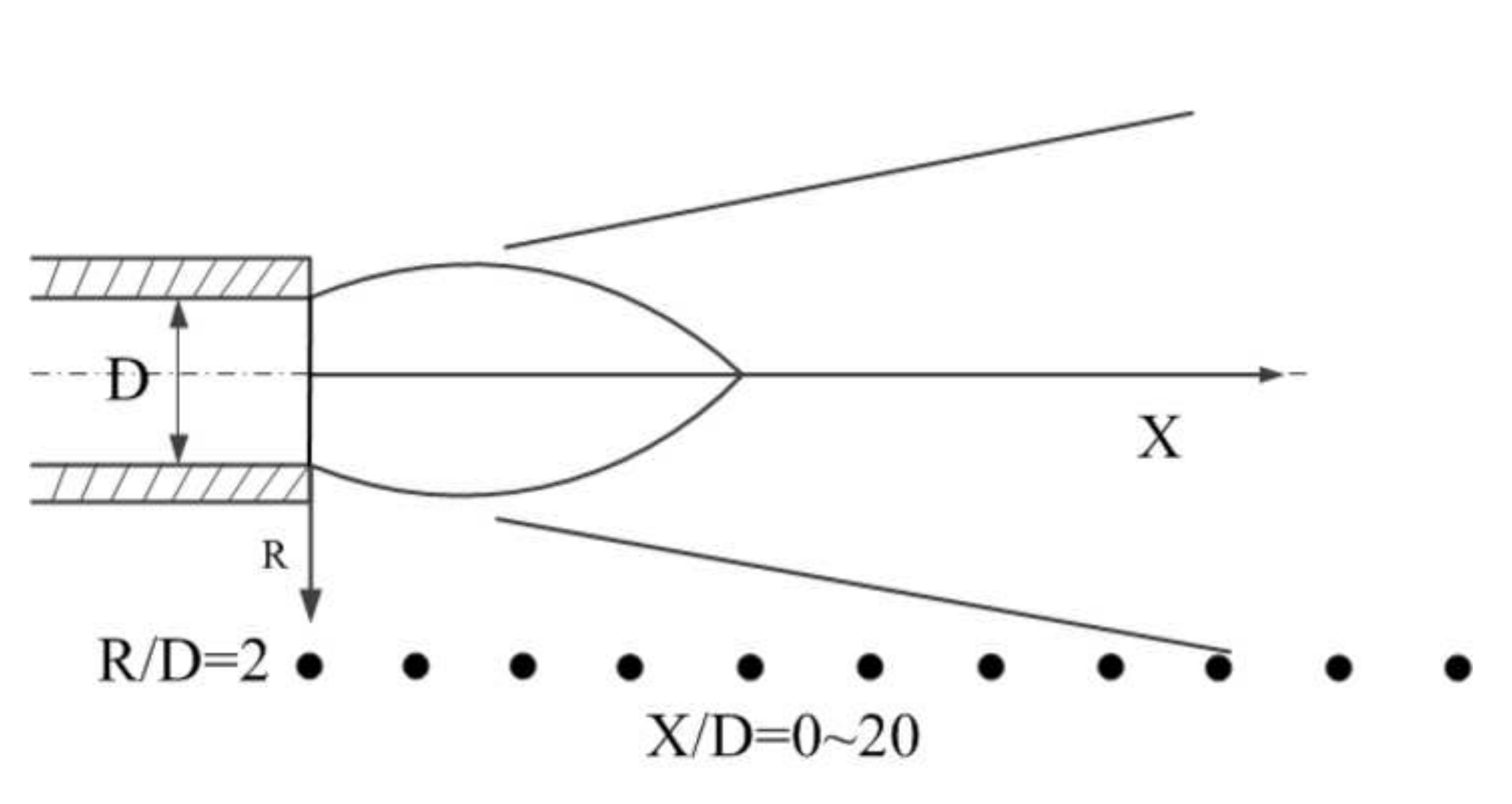

$$
\mathrm{X} / \mathrm{D}=0 \sim 20
$$

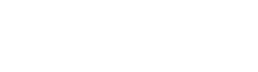
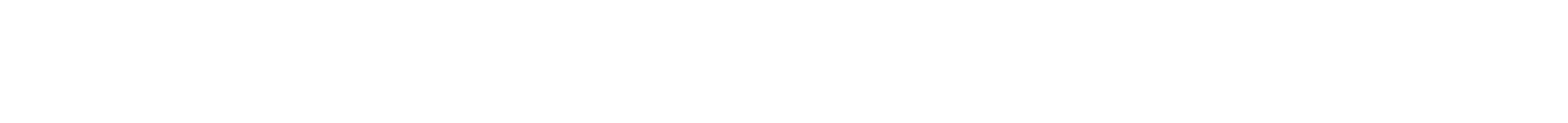

$$
\mathrm{X} / \mathrm{D}=0-20
$$

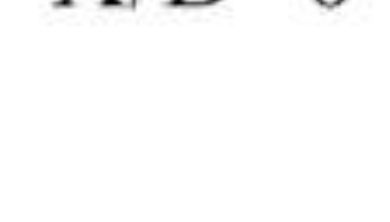

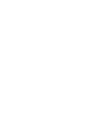

(2n)

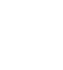$$
\text { ( }
$$

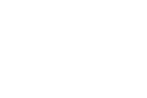

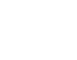




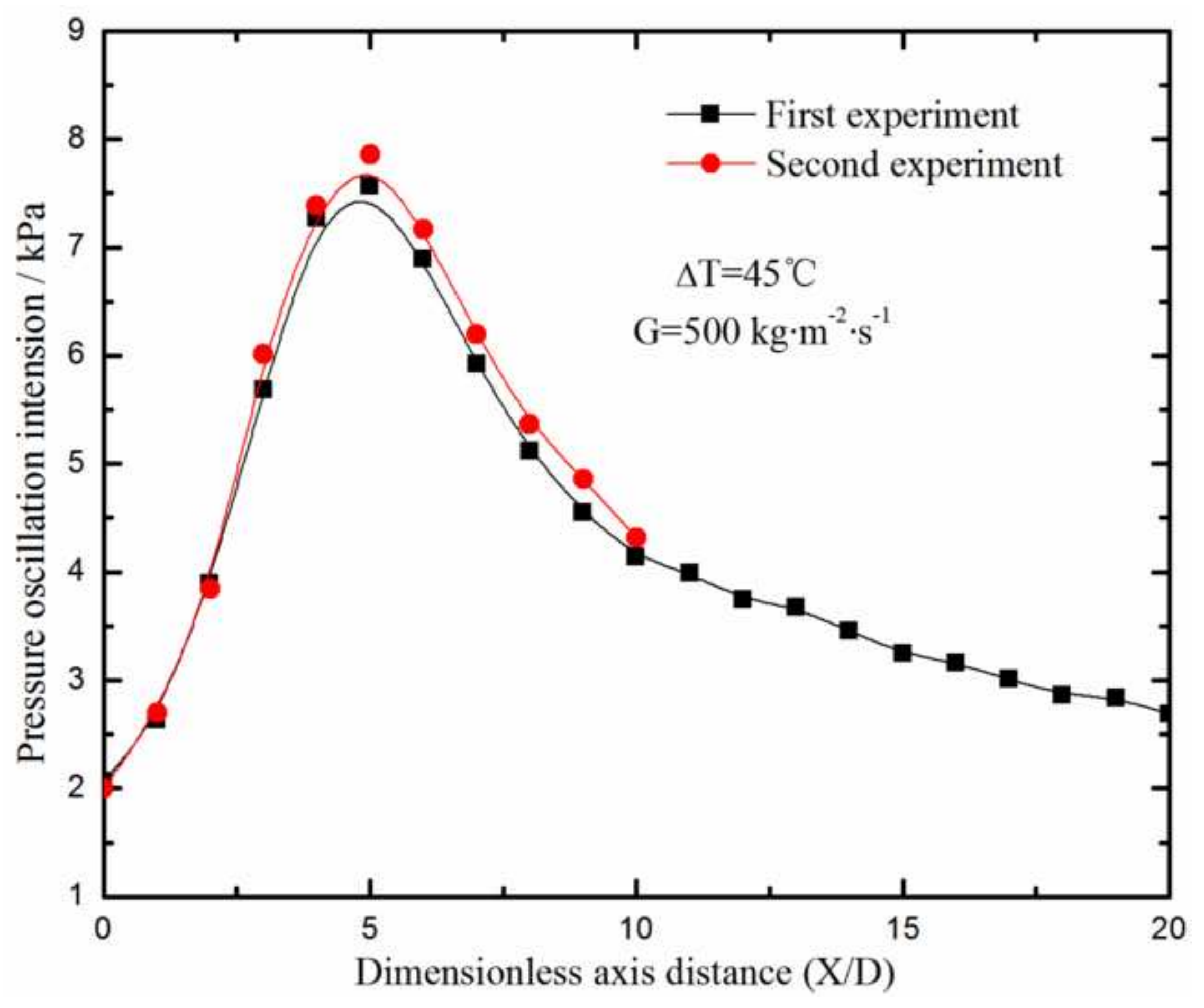



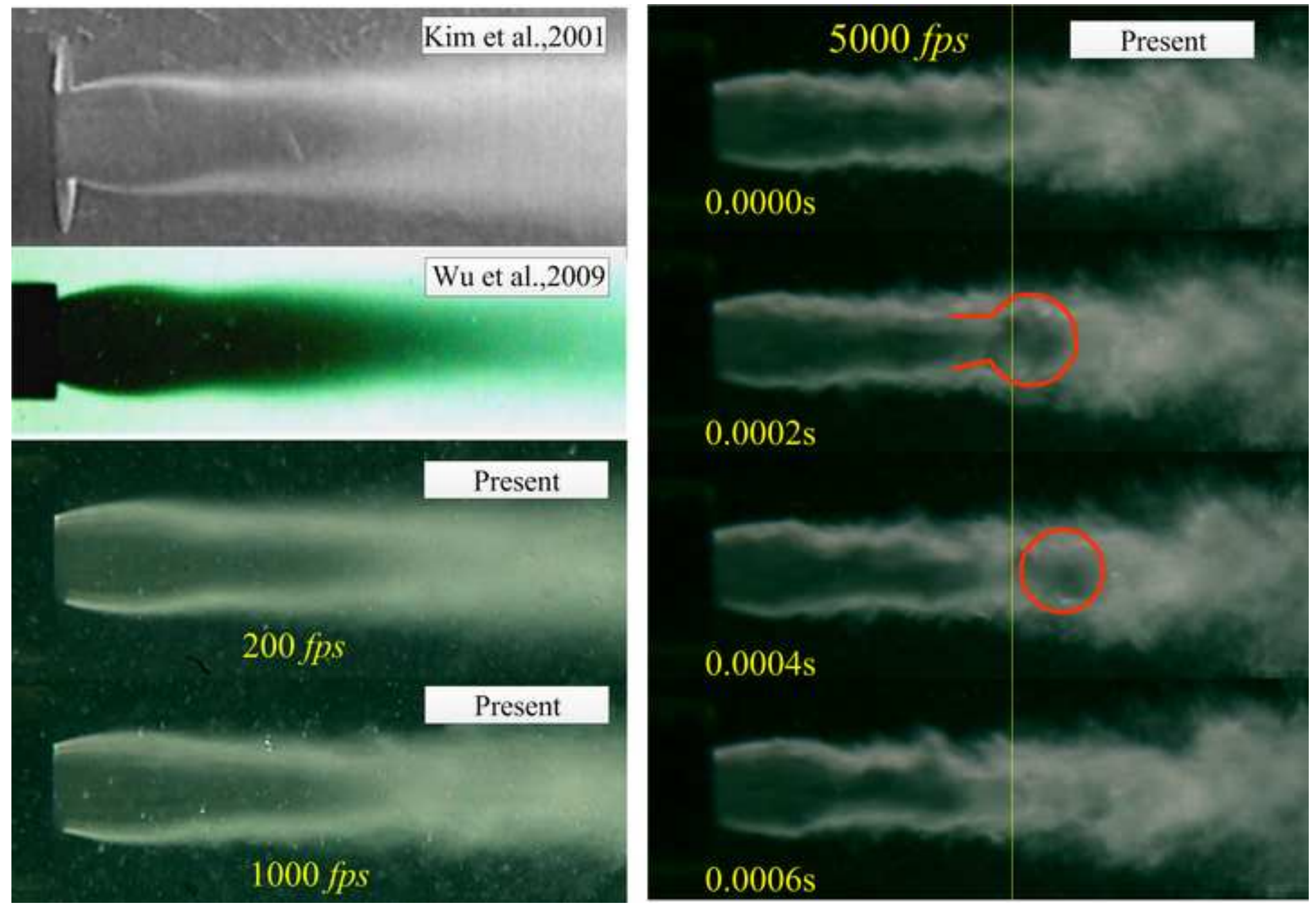

Wu et al.,2009

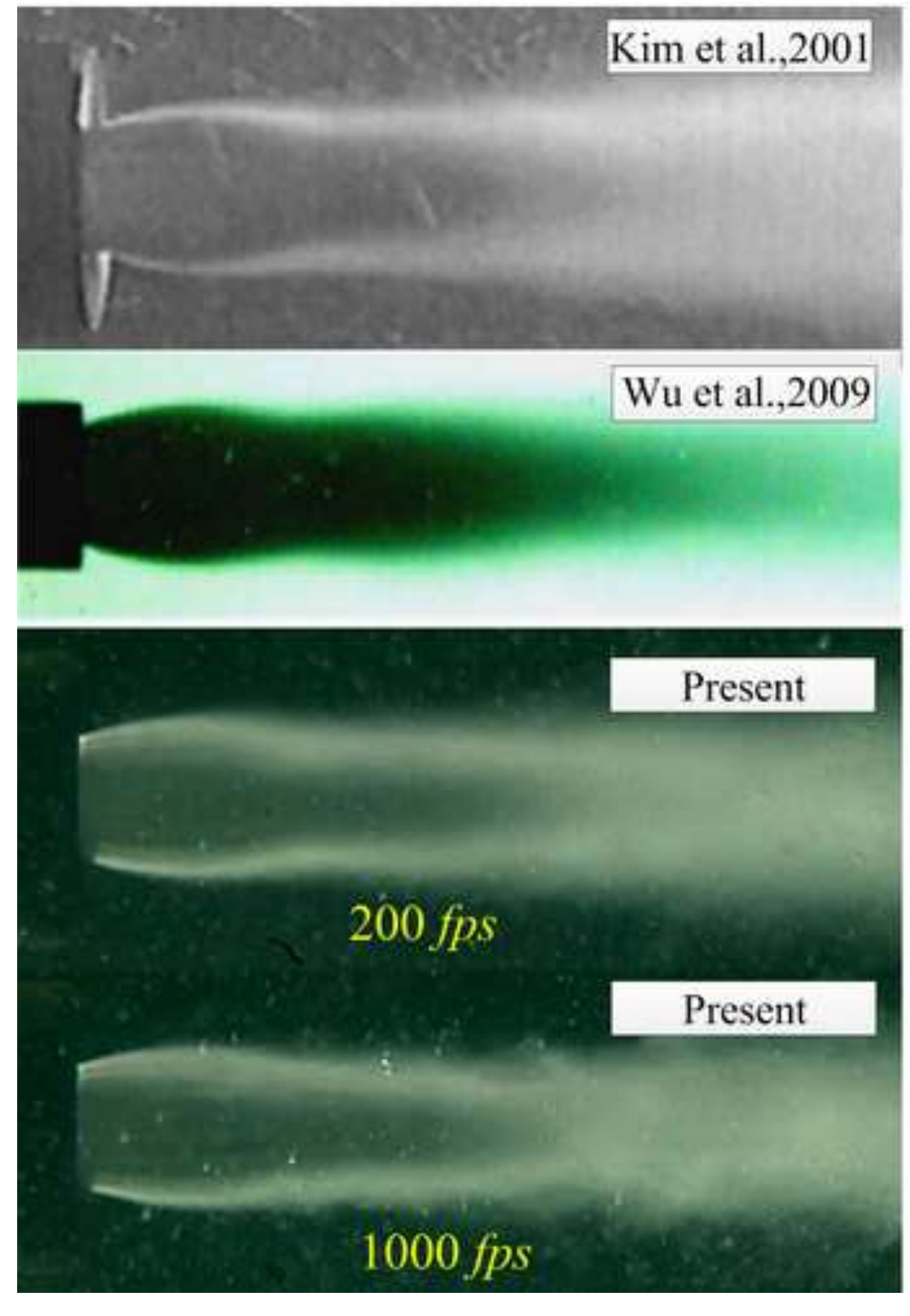

$0.0006 \mathrm{~s}$

\section{$0.0004 \mathrm{~s}$}

\section{$0.0002 \mathrm{~s}$}

\subsection{6}




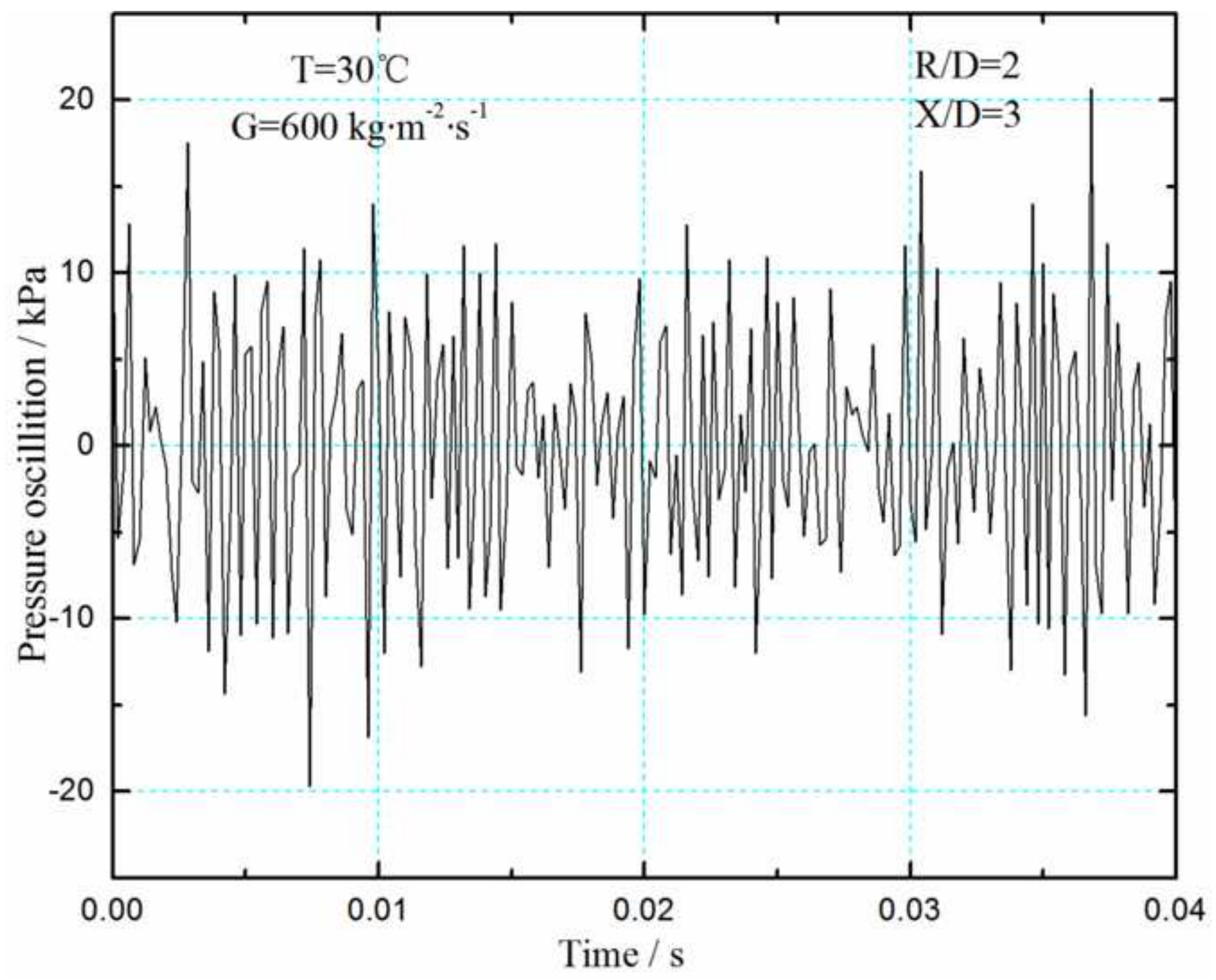




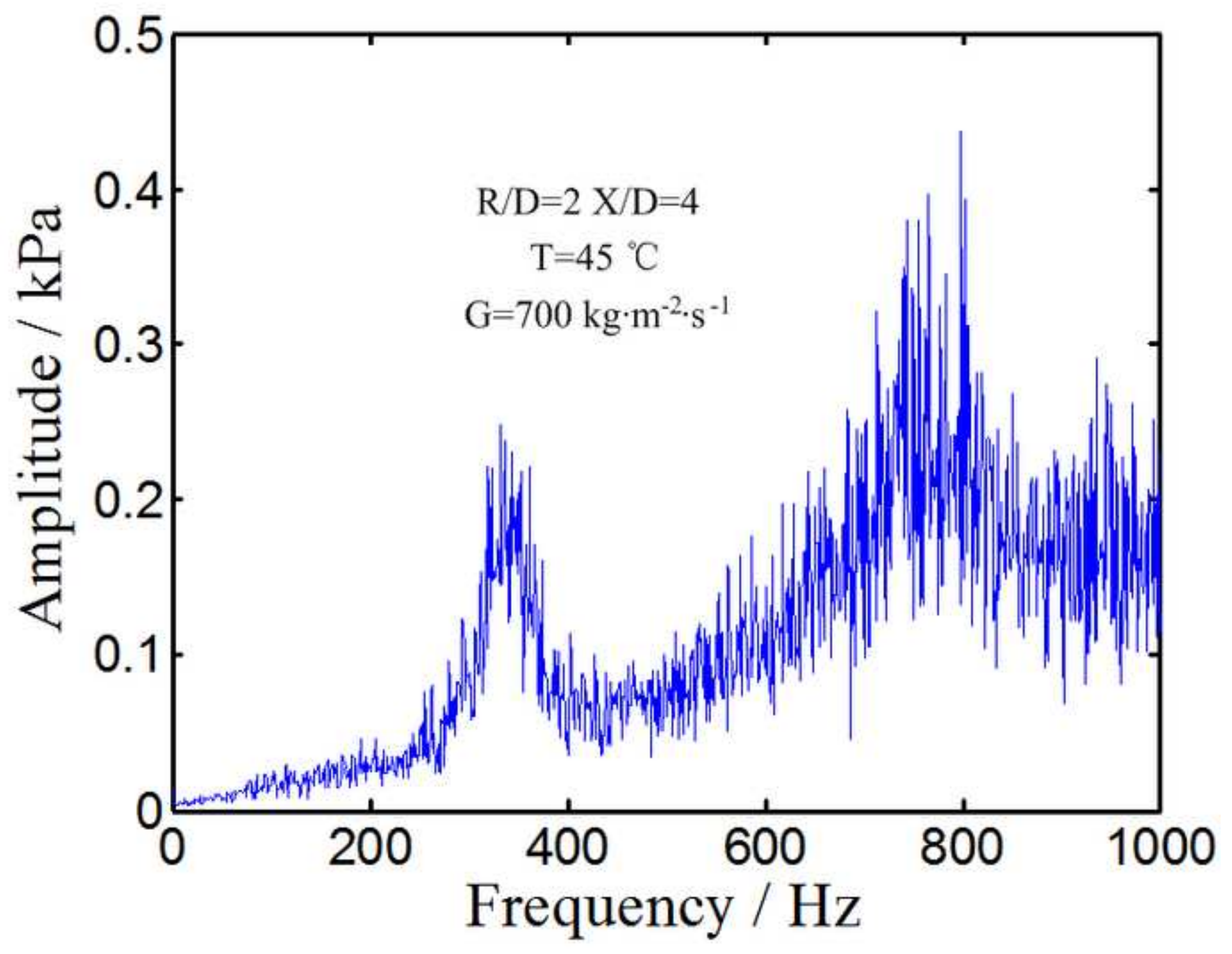



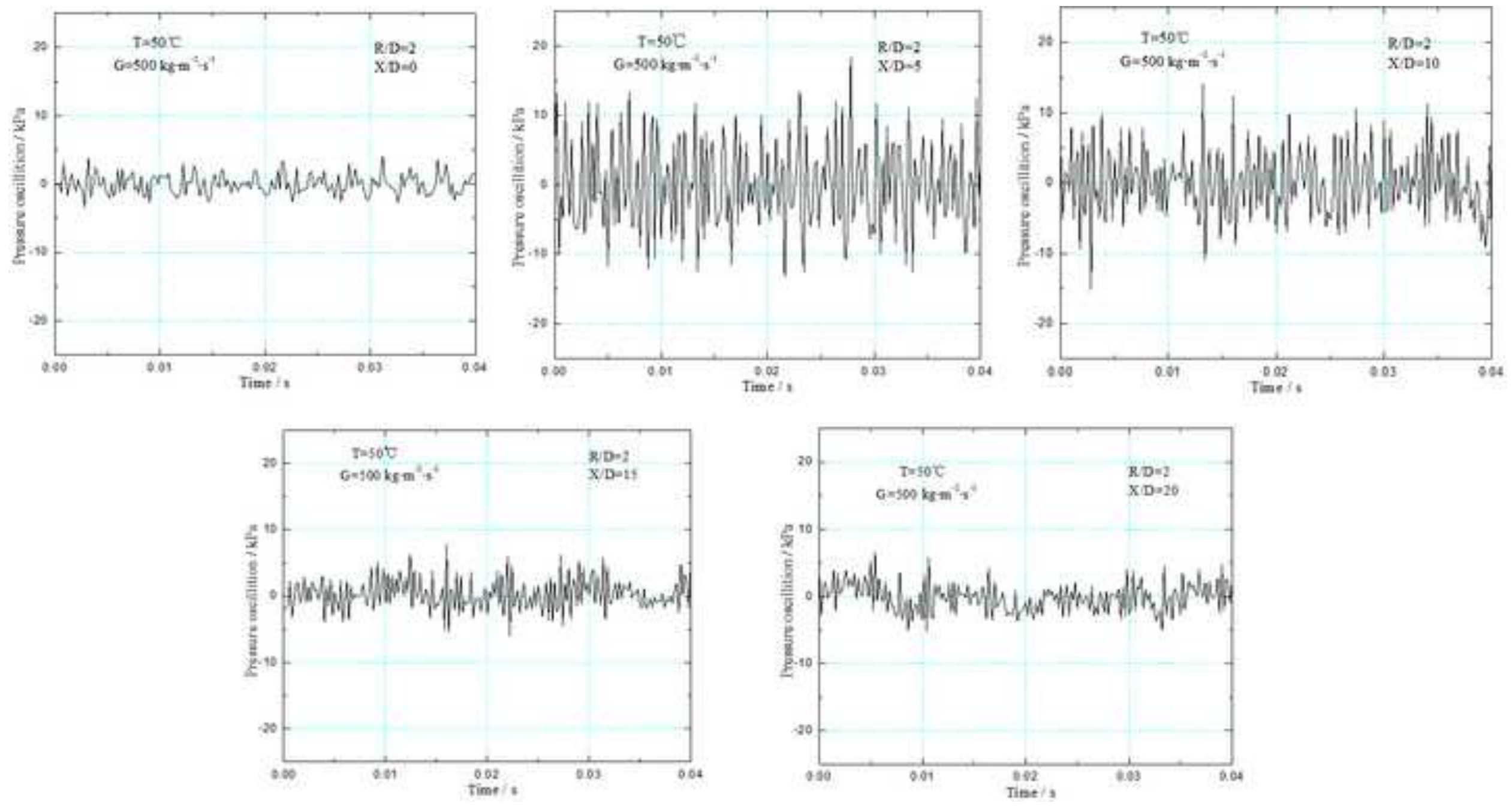


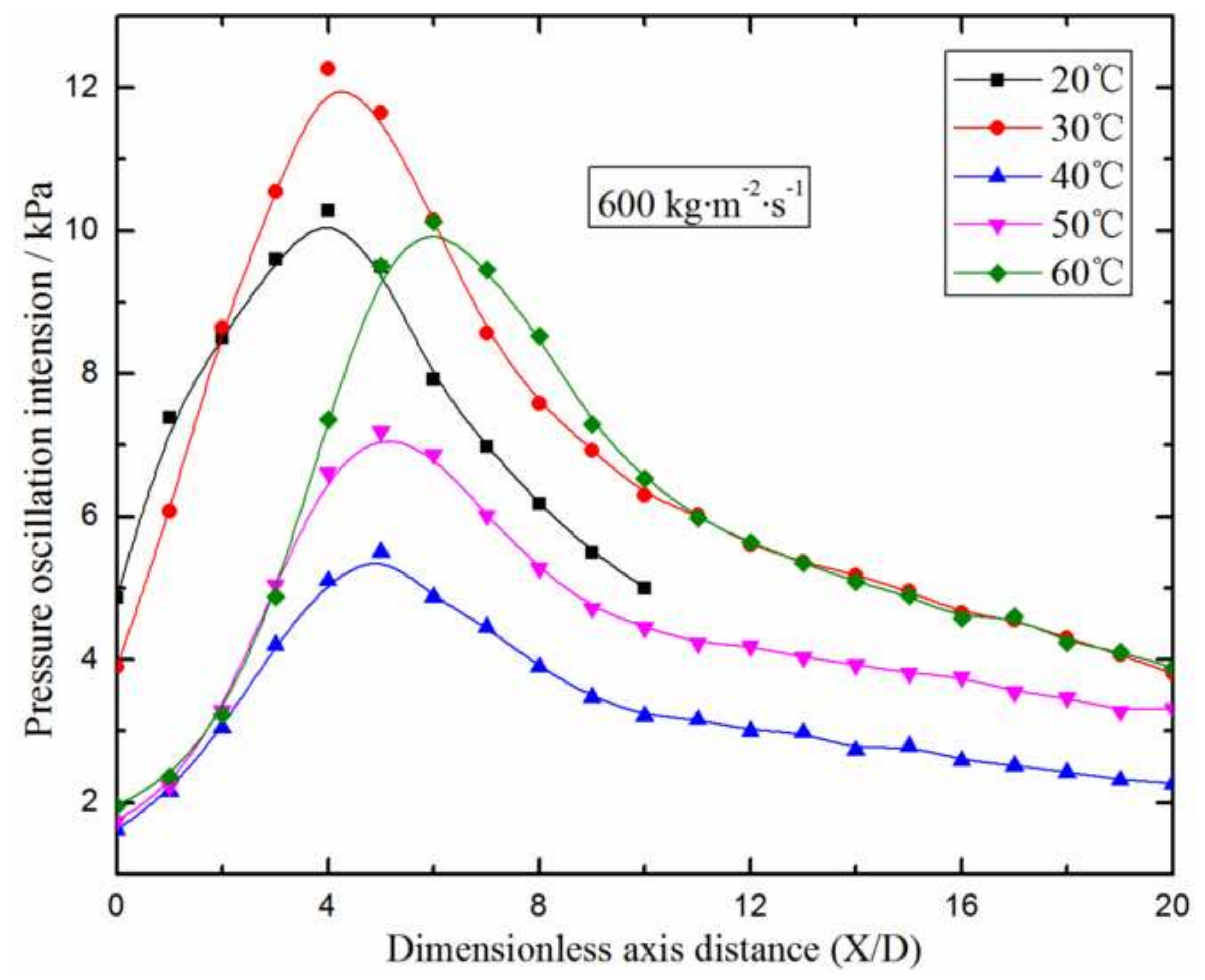




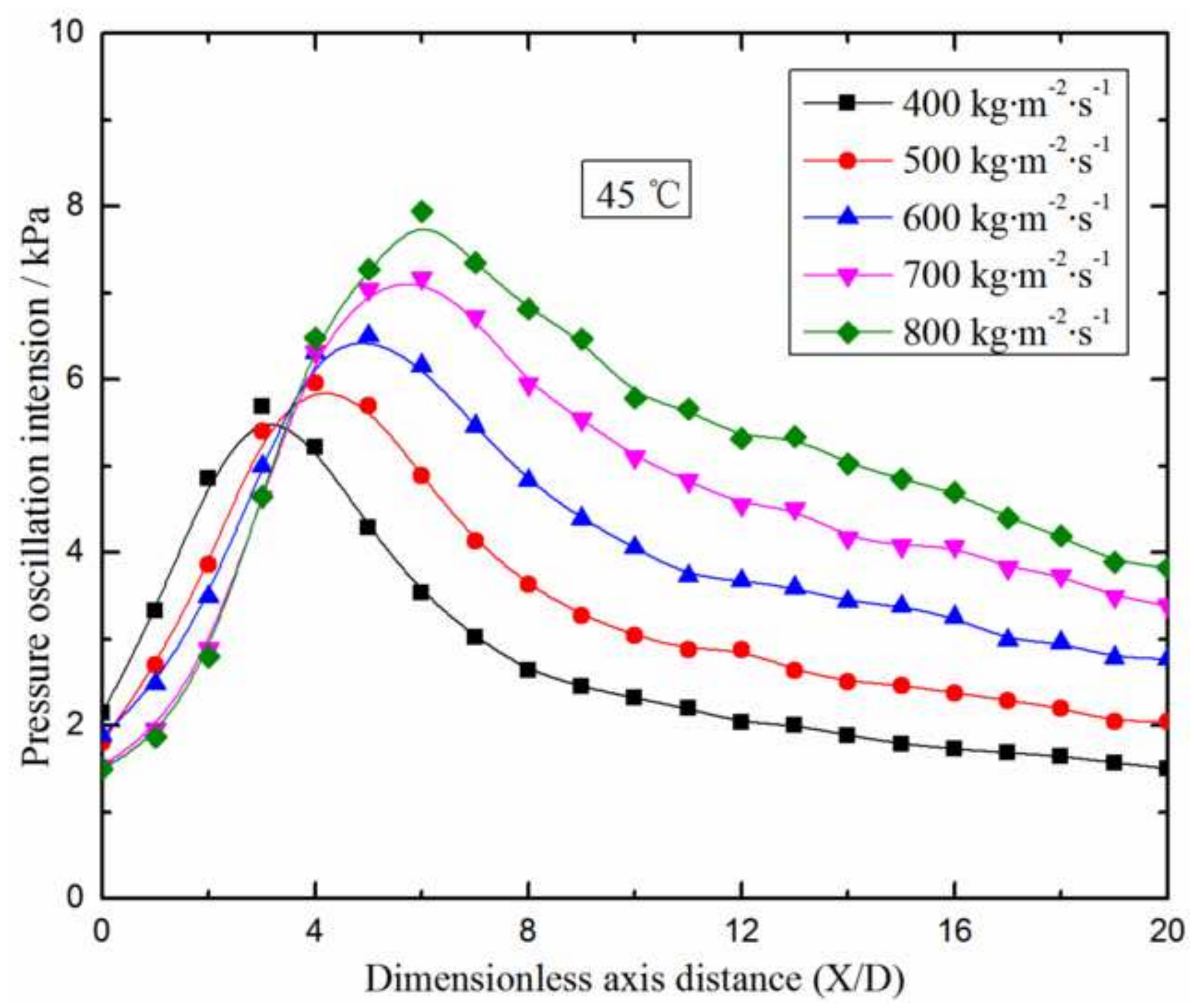




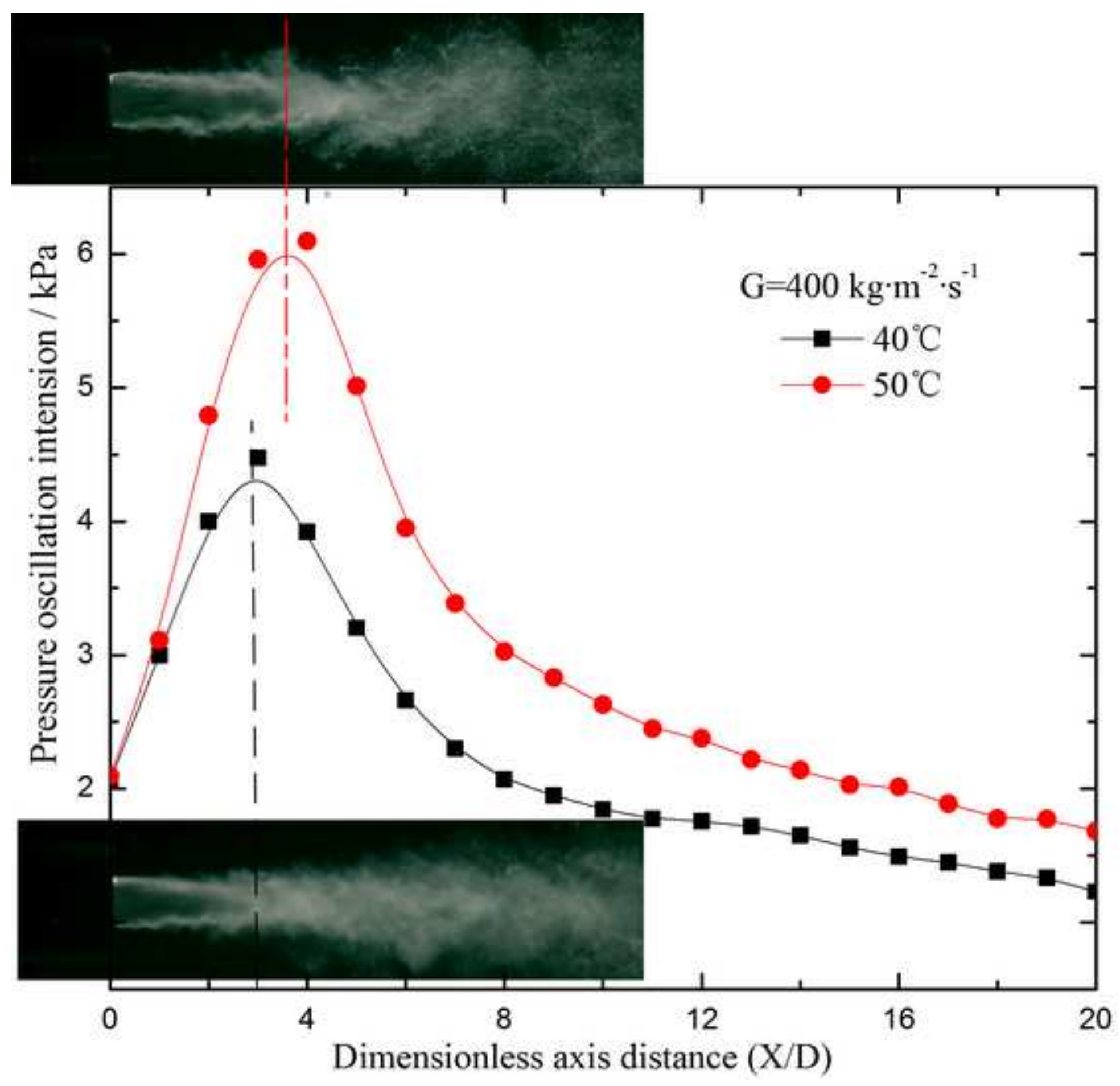




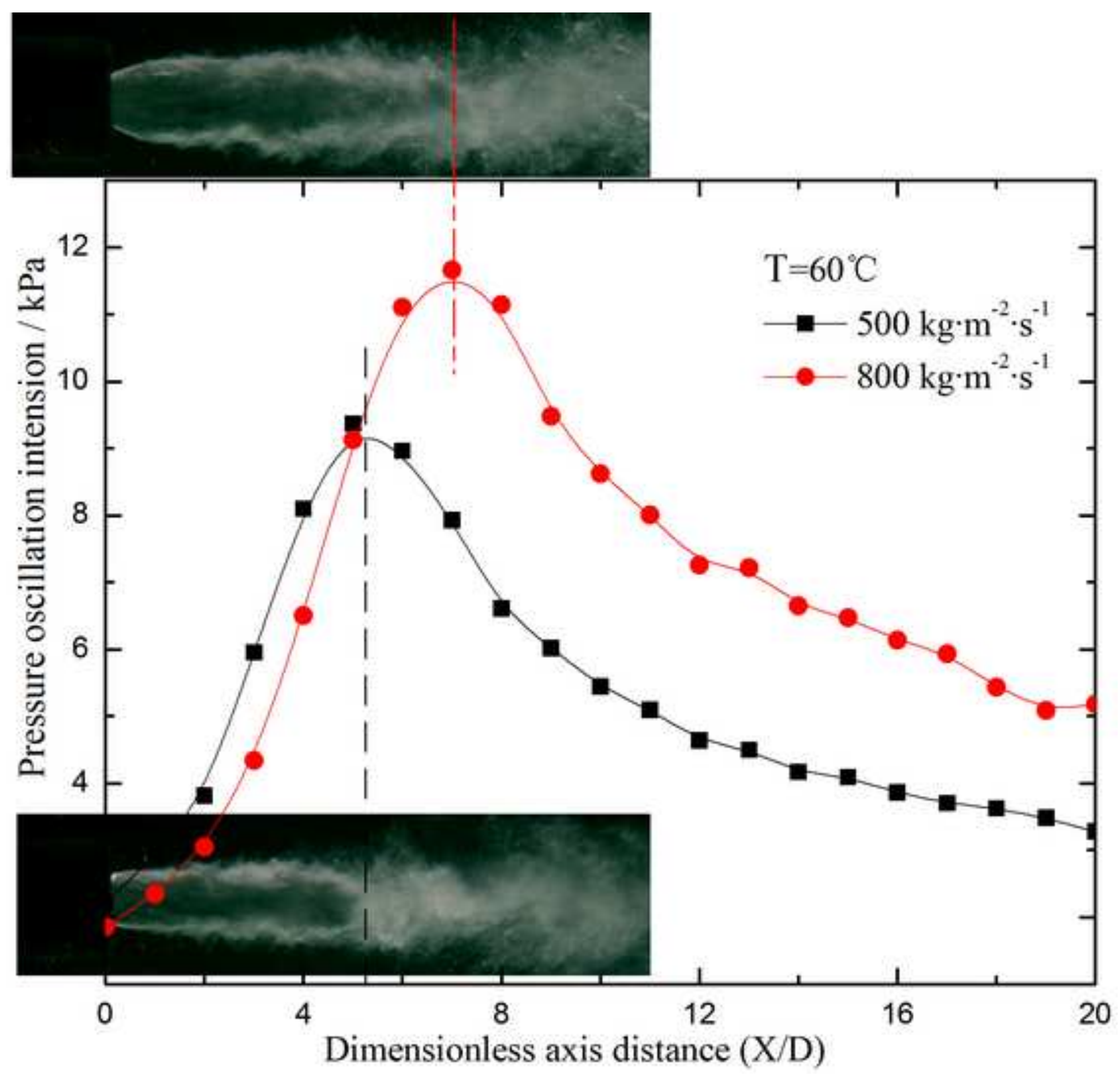




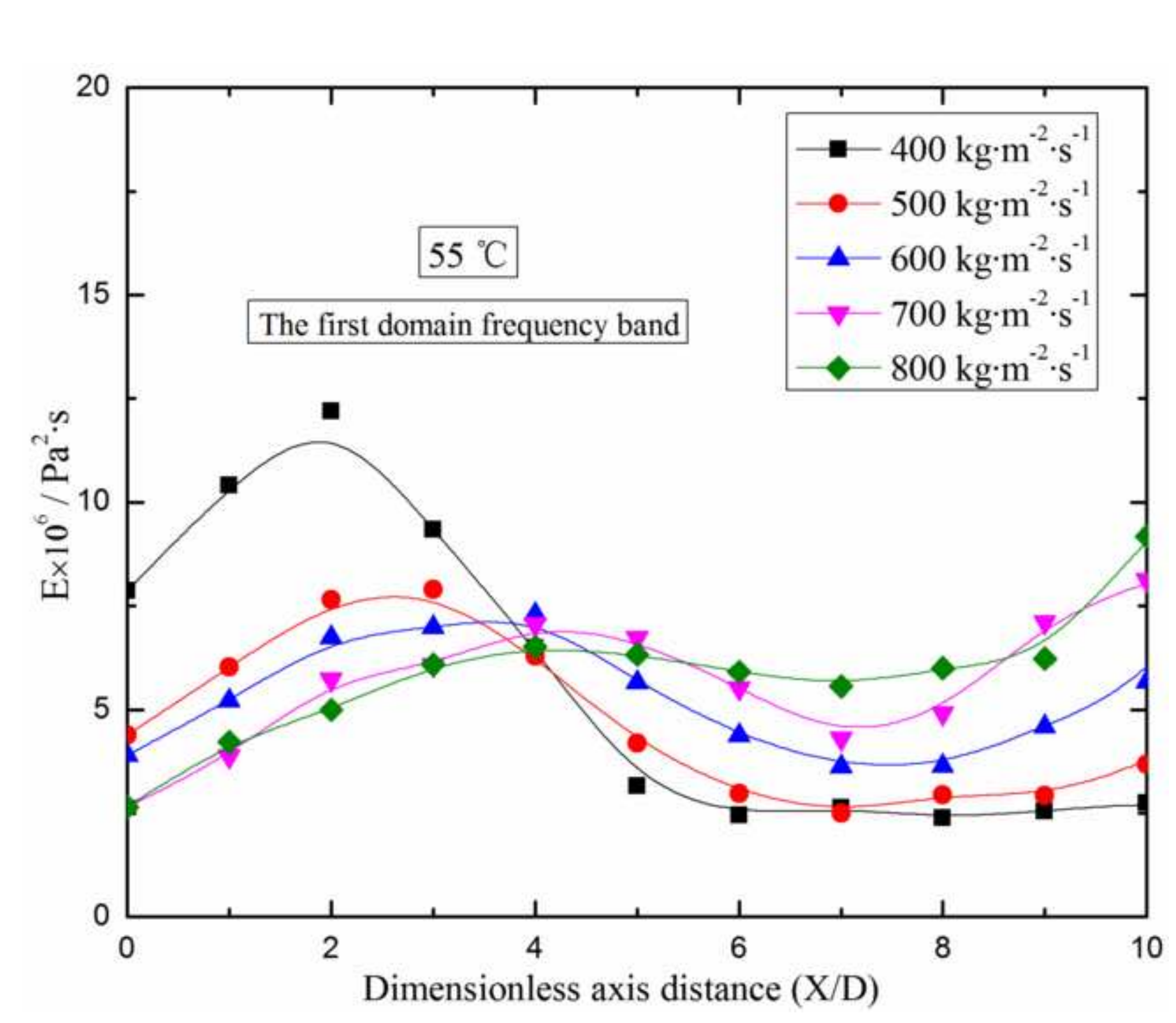




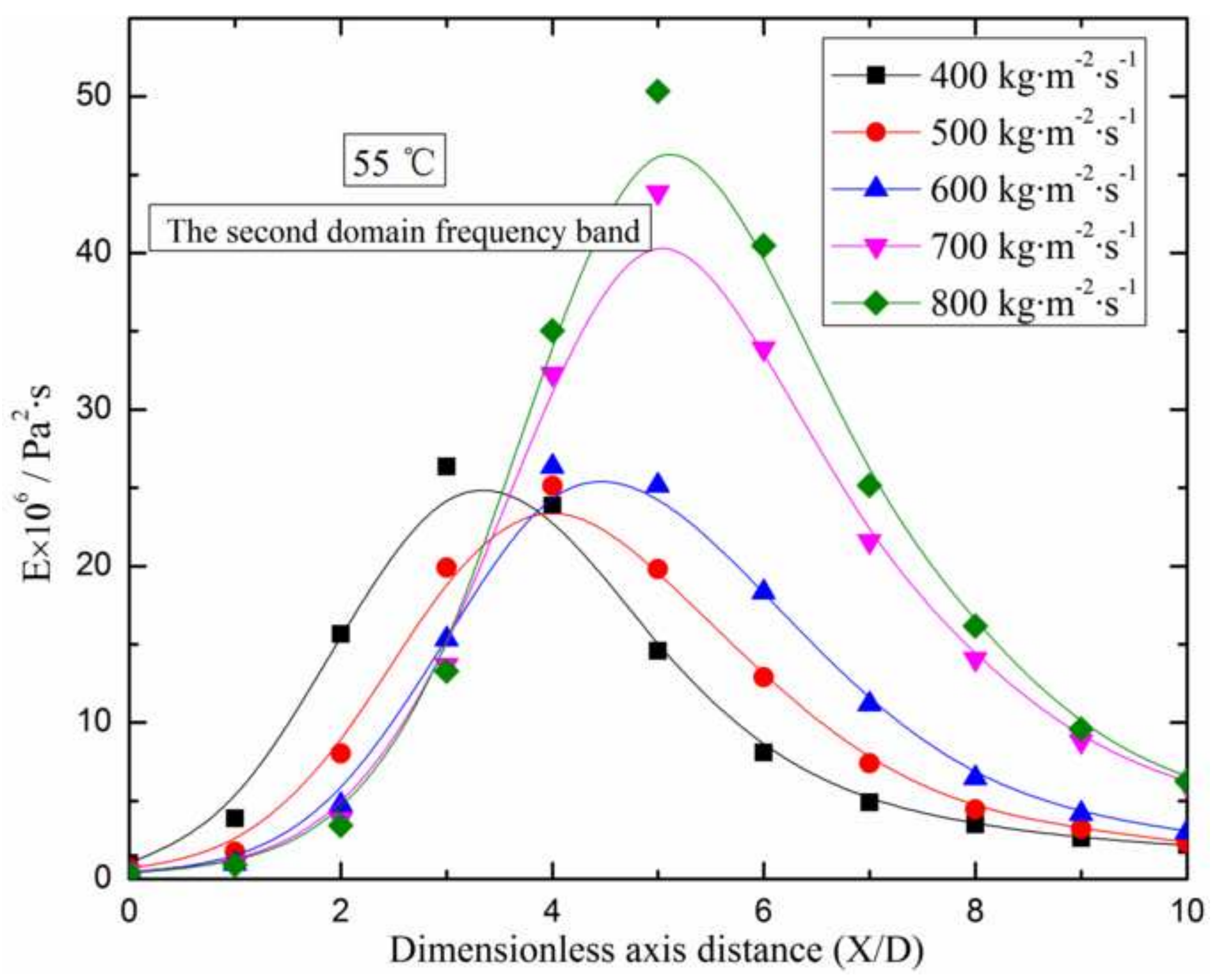




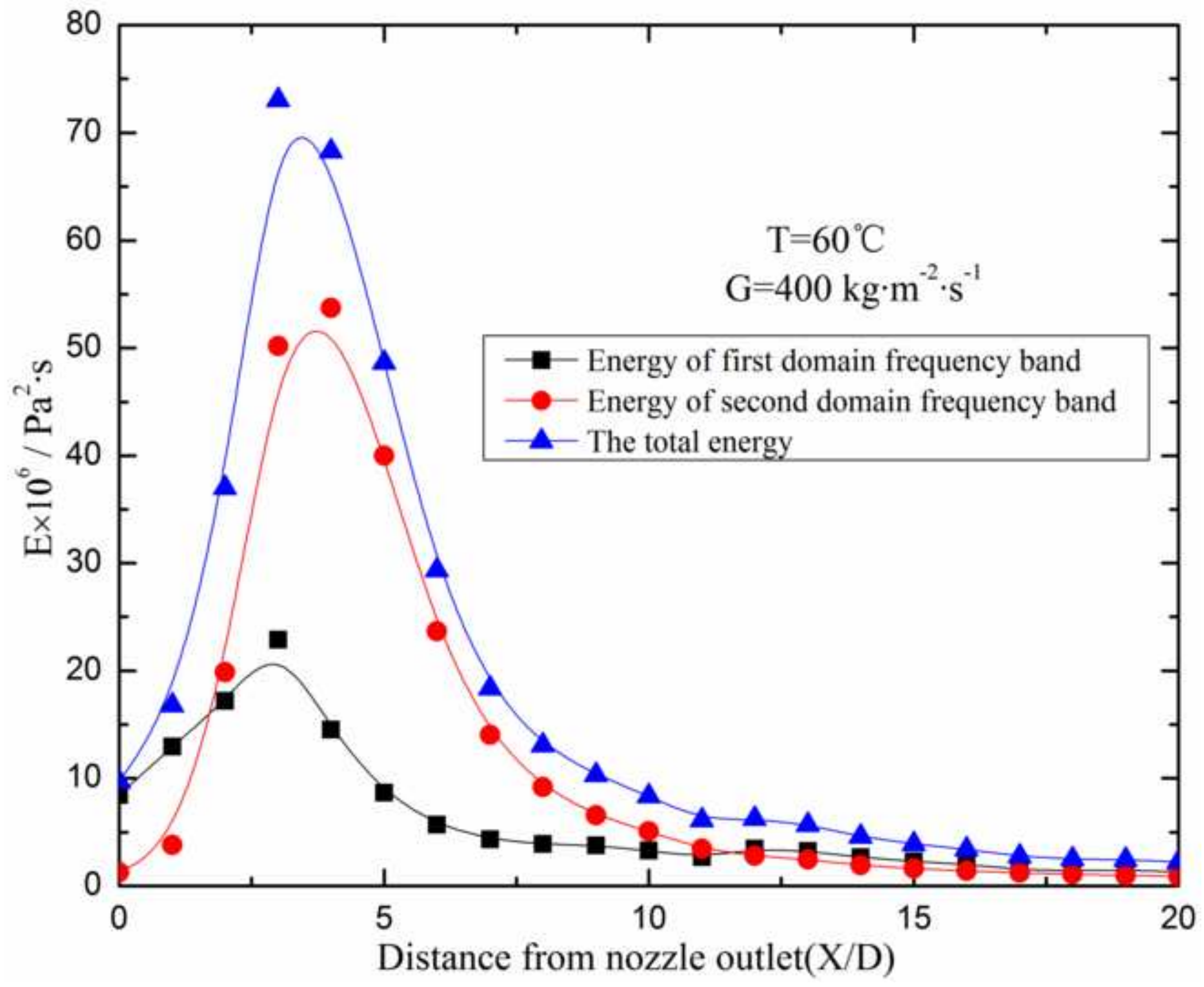




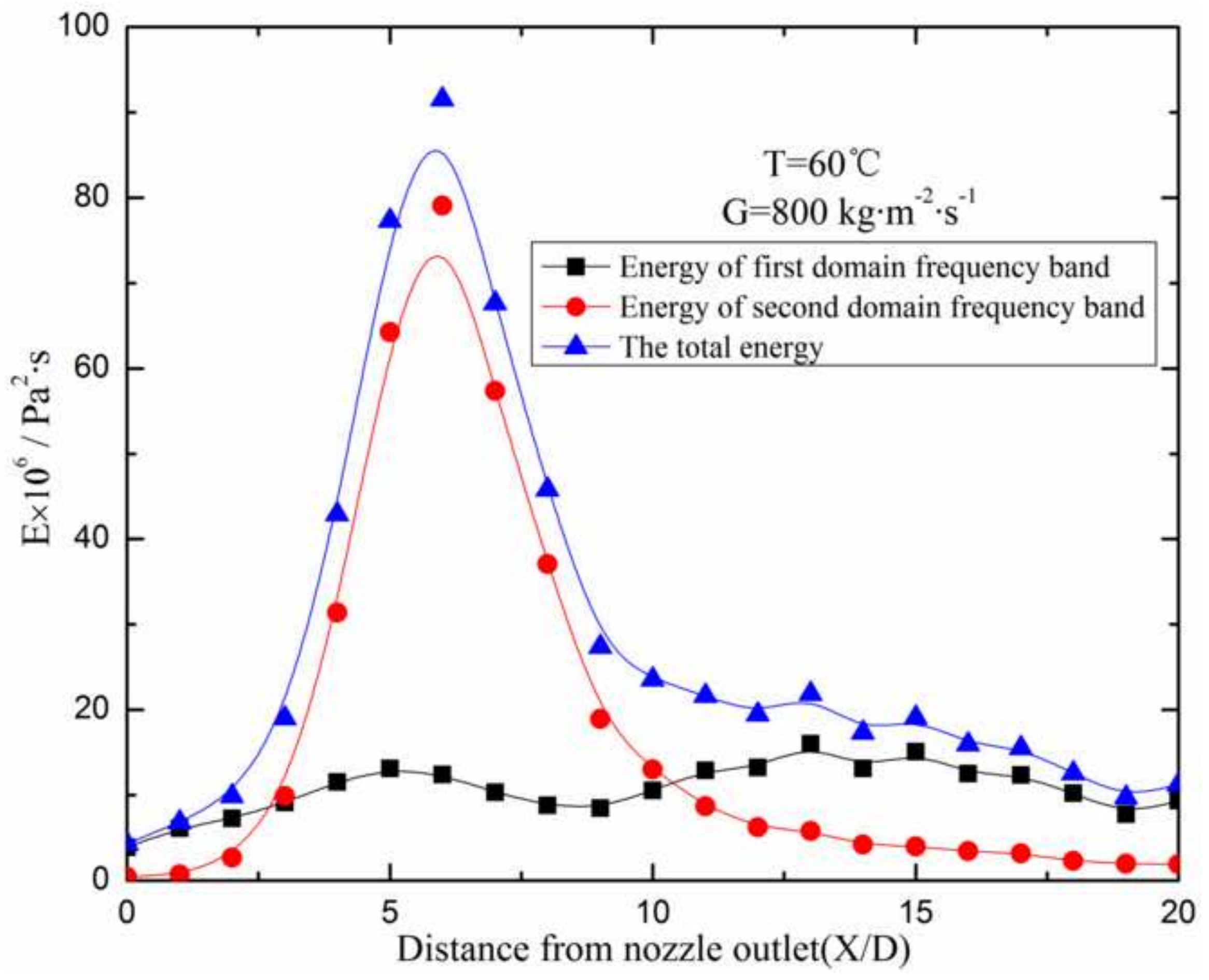




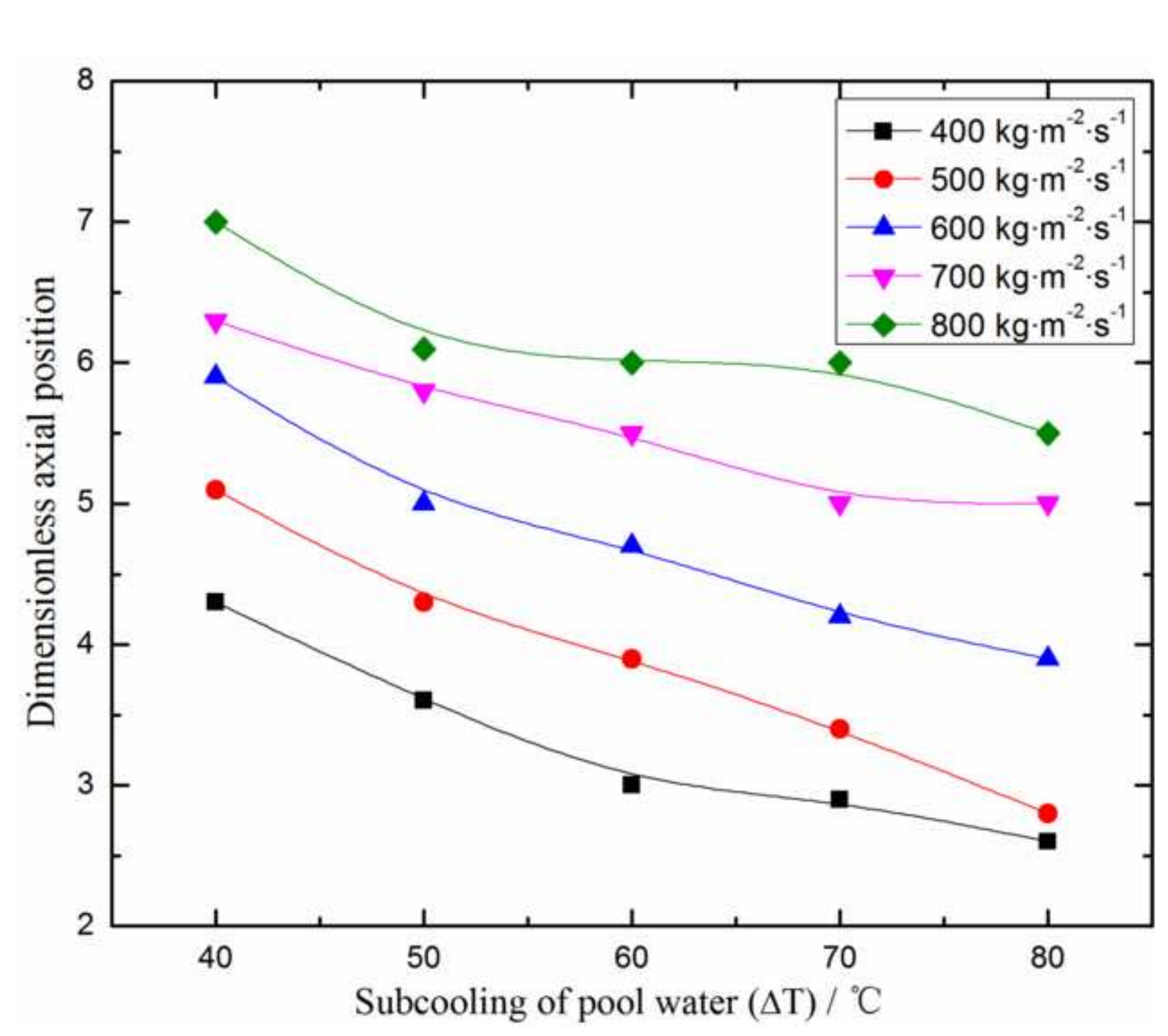




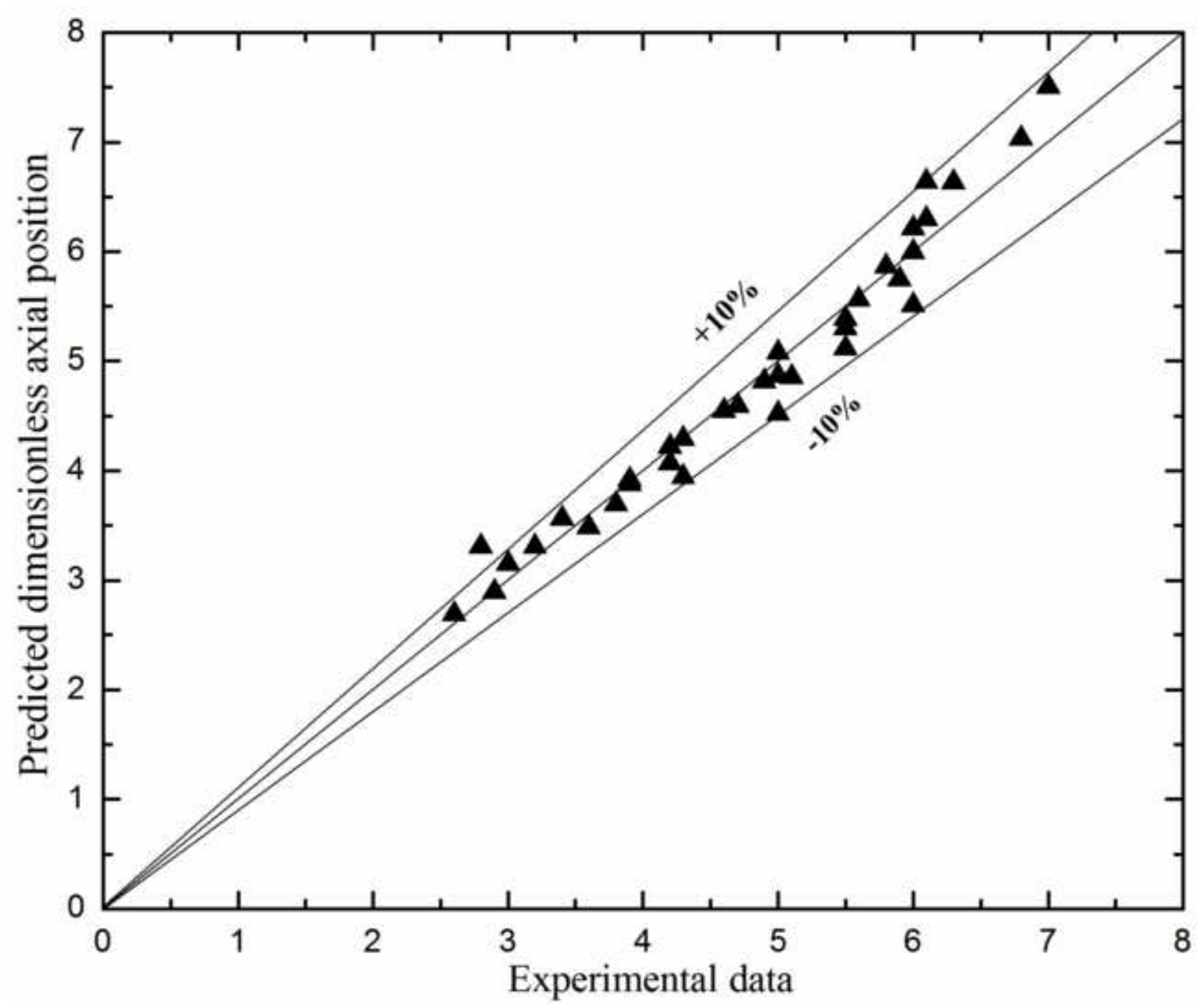




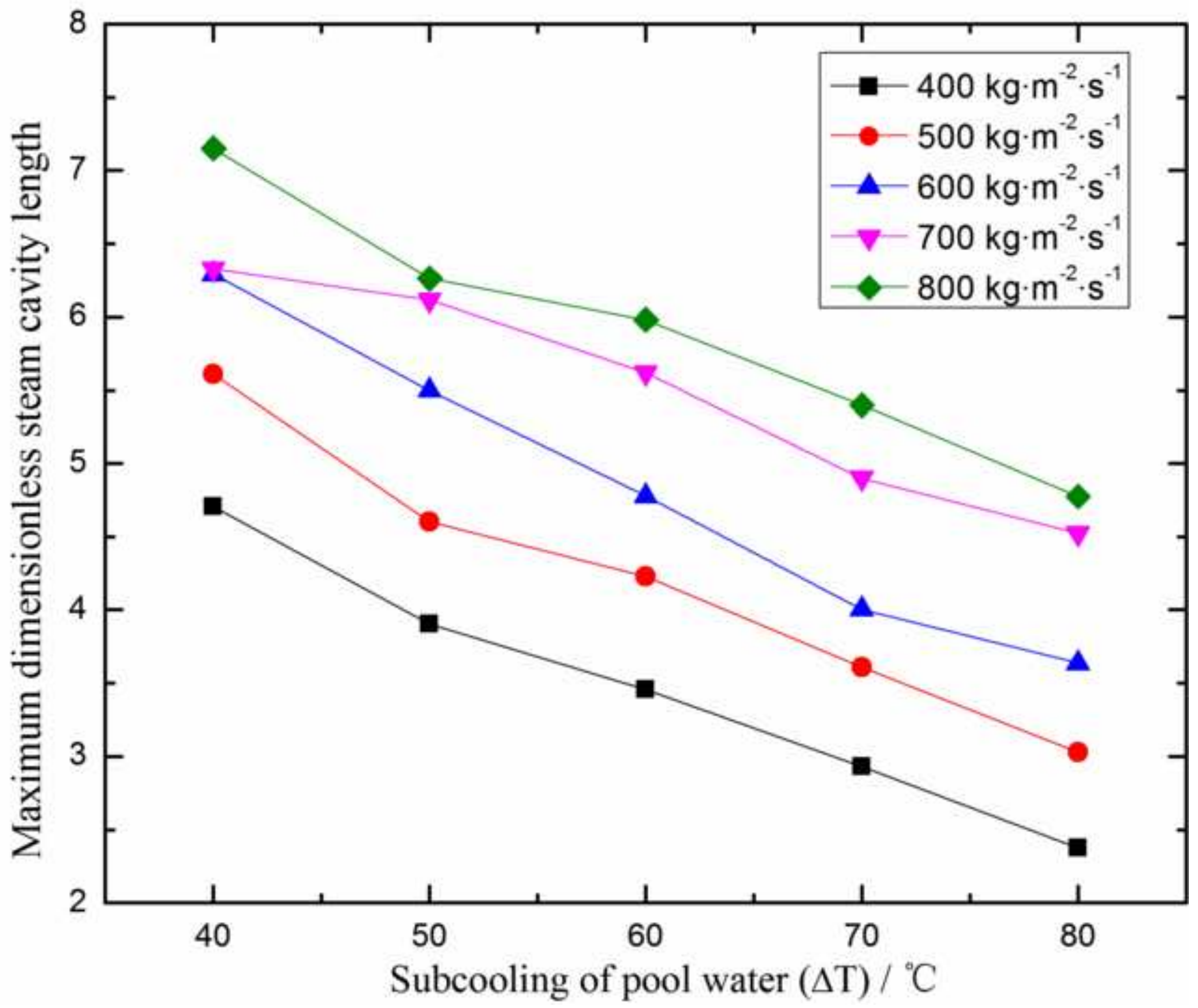




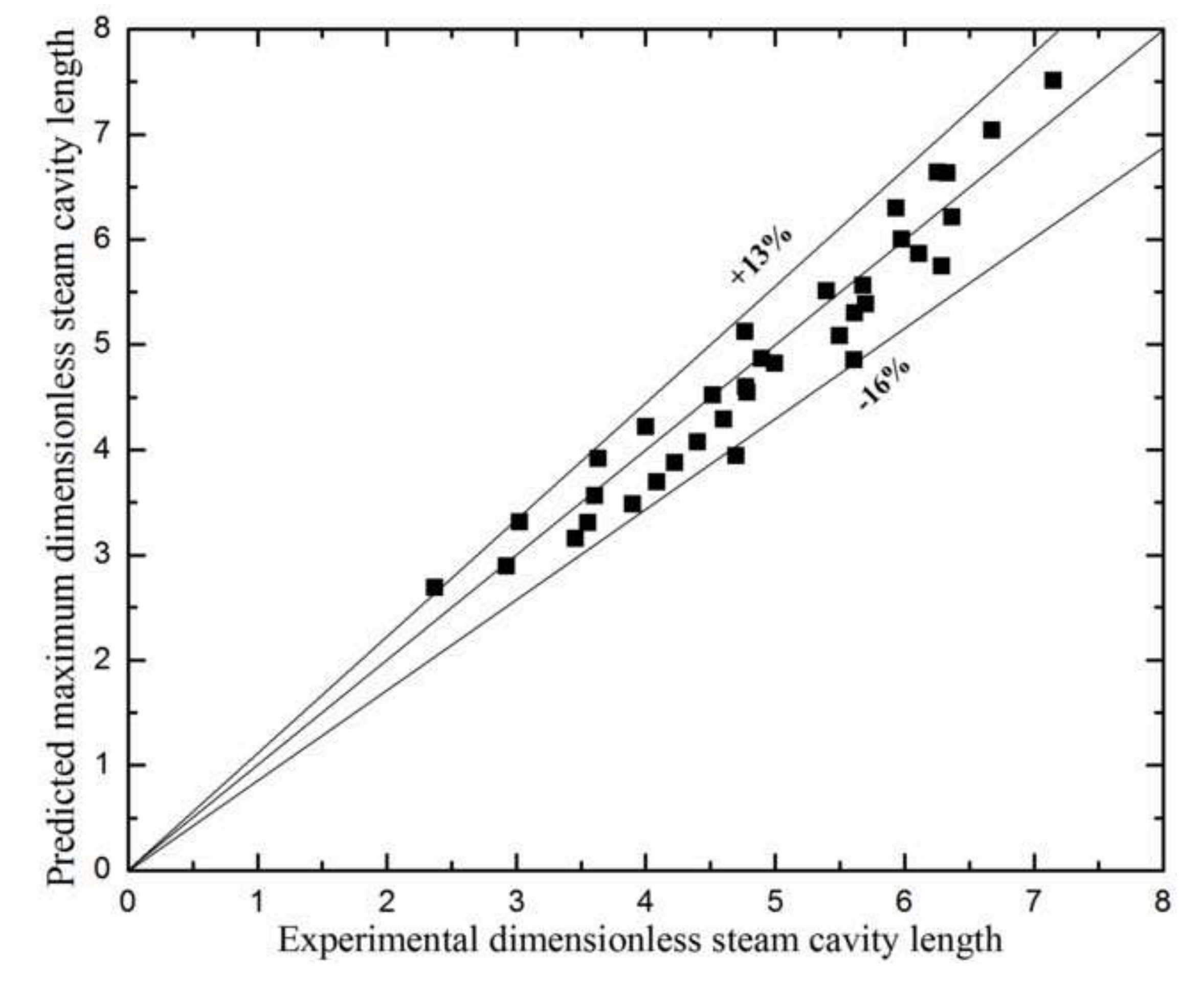

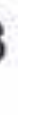

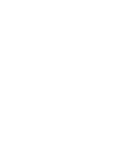

. 
Table 1 Test conditions in experiment

\begin{tabular}{ccc}
\hline Parameters & value & units \\
\hline Steam mass flux, $\mathrm{G}$ & $400-800$ & $\mathrm{~kg} \cdot \mathrm{m}^{-2} \cdot \mathrm{s}^{-1}$ \\
Water temperature $\mathrm{T}$ & $20-60$ & ${ }^{\circ} \mathrm{C}$ \\
Nozzle diameter, D & 8,10 & $\mathrm{~mm}$ \\
Submerged depth of & 500 & $\mathrm{~mm}$ \\
nozzle, $\mathrm{H}$ & 0.101 & $\mathrm{MPa}$ \\
Ambient pressure, $\mathrm{P}_{\mathrm{b}}$ & & \\
\hline
\end{tabular}

